\title{
A Survey of Sybil Attack Countermeasures in loT-based Wireless Sensor Networks
}

\author{
Akashah Arshad ${ }^{1}$, Zurina Mohd Hanapi ${ }^{\text {Corresp., } 1 \text {, Shamala K. Subramaniam }}{ }^{1}$, Rohaya Latip $^{1}$ \\ ${ }^{1}$ Faculty of Computer Science and Information Technology, Universiti Putra Malaysia, UPM Serdang, Selangor Darul Ehsan, Malaysia \\ Corresponding Author: Zurina Mohd Hanapi \\ Email address: zurinamh@upm.edu.my
}

Wireless sensor networks (WSN) have been among the most prevalent wireless innovations over the years, the exciting new Internet of Things (IOT) applications. IoT based WSN integrated with Internet Protocol (IP) allows any physical objects with sensors to be connected ubiquitous and send real-time data to the server connected to the Internet gate. Security in WSN remains an ongoing research trend that falls under the IoT paradigm. WSN node deployed in a hostile environment is likely to open security attacks such as Sybil due to its distributed architecture and network contention implemented in the routing protocol. In a Sybil attack, an adversary illegally advertises several false identities or a single identity that may occur at several locations called Sybil nodes. Therefore, in this paper, we give a survey of the most assuring methods up to date to defend from the Sybil attack. The Sybil attack countermeasure includes encryption, trust, received signal indicator (RSSI), encryption and swarm intelligence. Specifically, we survey different methods, along with their advantages and disadvantages, to mitigate the Sybil attack. We discussed the lesson learned and the future avenues of study and open issues in WSN security analysis. 
1 A Survey of Sybil Attack Countermeasures in IoT based

2 Wireless Sensor Networks

3

4

5

6

7

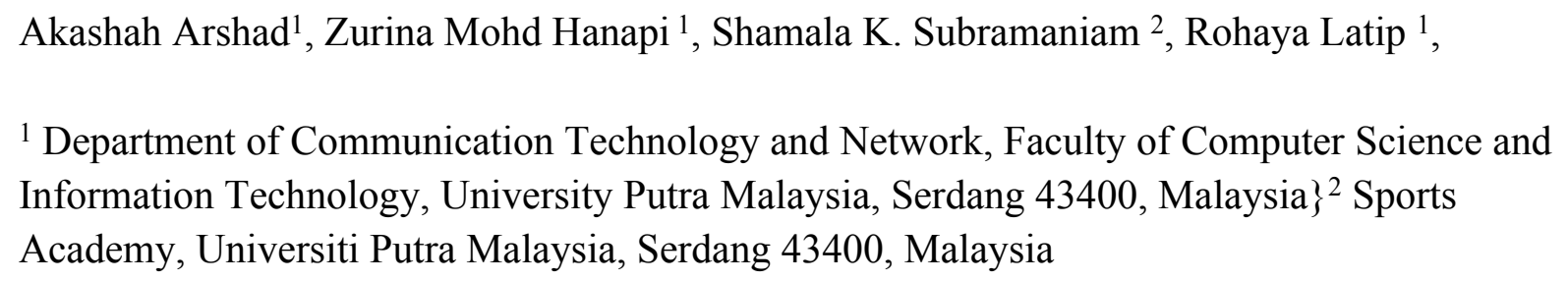

\section{ABSTRACT}

Wireless sensor networks (WSN) have been among the most prevalent wireless innovations over the years, the exciting new Internet of Things (IoT) applications. IoT based WSN integrated with Internet Protocol (IP) allows any physical objects with sensors to be connected ubiquitous and send real-time data to the server connected to the Internet gate. Security in WSN remains an ongoing research trend that falls under the IoT paradigm.

WSN node deployed in a hostile environment is likely to open security attacks such as Sybil due to its distributed architecture and network contention implemented in the routing protocol. In a Sybil attack, an adversary illegally advertises several false identities or a single identity that may occur at several locations called Sybil nodes.

Therefore, in this paper, we give a survey of the most assuring methods up to date to defend from the Sybil attack. The Sybil attack countermeasure includes encryption, trust, received signal indicator (RSSI), encryption and swarm intelligence.

Specifically, we survey different methods, along with their advantages and disadvantages, to mitigate the Sybil attack. We discussed the lesson learned and the future avenues of study and open issues in WSN security analysis.

\section{INTRODUCTION}

The Internet of Things ( IoT) gained universal acceptance due to many applications for personal use and the community. IoT represented a collection of "Things" or embedded devices connected using various wireless technologies such as private and public networks (Atzori, Iera, \& 
Morabito, 2010). Based on the application domain, IoT applications are classifiable into five groups, for example, health care (Zeb, Islam, Zareei, Mamoon, \& Mansoor, 2016); Ambarkar \& Shekokar, 2020), environmental (Kumari \& Sahana, 2019; Behera et al., 2020; Zhuang et al., 2019; Jawad, Nordin, \& Gharghan, 2017), smart city (Santos, Jimenez, \& Espinosa, 2019; Luo, 2019), commercial (G. Li et al., 2018; Khanna \& Tomar, 2016), IoT based robotic (Roy Chowdhury, 2017) and industry (M. Zhu et al., 2020).

Wireless sensor networks (WSNs) are essential subsets of the Internet of Things (IoT) that have emerged as a core technology for a variety of data-centric applications. Almost all IoT network concepts are derived from WSNs. Both terms can be confusing at times, and they have a lot of similarities and differences between IoT and WSN (Pundir, Wazid, \& Singh, 2020). IoT based WSN integrated with Internet Protocol (IP) allows any physical objects with sensors to be connected ubiquitous and send real-time data to the server connected to the internet gateway. Sensor data is relayed to the base station and is saved in the cloud for future access (Ala'Anzy \& Othman, 2019; Sheron, Sridhar, Baskar, \& Shakeel, 2020). IoT-based WSN devices are powered by batteries that later can be replaced, which poses a significant challenge to application designers. To address these constraints in an IoT-based WSN, significant research has been conducted on managing network power consumption. Most existing research focuses on extending the IoT network lifetime. The purpose of WSN is to gather data from the sensor node in the predetermine or random location and transmit the sensed data back to the base station.

The cumulative confirmed COVID-19 cases between 22 January and 12 October 2020 has reached 38,789,204 confirmed cases and has resulted in 1,095,097 deaths globally, as reported by WHO (2020). As a result, the need for monitoring systems is in great demand. The health of COVID-19 patients will be monitor continuously in an isolated room. Six per cent of them need to warded in the Intensive Care Unit (ICU) to save their lives, as reported by ElRashidy et al. (2020).

Gupta et al. (2020) foresee that smart sensors, actuators, devices, and data-driven applications can enable smart connected communities to strengthen the nations' health and economic postures to combat current Covid-19 and future pandemics efficiently. Flying drones regulated the quarantine and wearing of masks for public surveillance. Indoor isolation is made more accessible by robots and digital assistants. With the help of aware IoT devices, it is possible to track the origins of epidemics and ensure that patients follow important medical advice., as highlighted by Fedele \& Merenda (2020). However, from a security perspective, IoT networks are prone to sensor-based attacks based on a recent survey conducted by Sikder et al. (2018). The authors also addressed IoT devices' vulnerability to sensor-based threats due to the lack of enough protection mechanisms to monitor the use of sensors by applications. An attack can be a launch to the IoT based health application use to monitor COVID-19 patient. This security attack can put the patient's life in danger, where the attacker can manipulate the medical IoT devices. 
79

80

81

82

83

84

85

86

87

88

89

90

91

92

93

94

95

96

97

98

99

100

101

102

103

104

105

106

107

108

109

110

111

112

113

114

115

116

117

Also, attackers can execute out local-scale attacks on individual critical devices that could include human life, such as the 2011 Stuxnet attack (Kushner, 2013), the late 2015 powergrid blackout of Ukraine (Dvorkin Yury, 2020), the 2015 Jeep Cherokee attack (Schneider David, 2015), the 2017 Brickerbot attack (Radware, 2017) and the 2018 Philips lightbulbs attack demonstration. In a world where every device is connected to IoT, these attacks have shown how catastrophic and diversified cybercrimes could be. Therefore, it is vital to detect Sybil attackers in WSN to prevent their malicious activities. In other words, Sybil attacks present a significant challenge for WSN, and improved defence mechanisms are required. We believe that the conducted survey work will help the researchers in this Sybil countermeasures in WSN.

The recent survey covers the existing countermeasures to mitigate the WSN and IoT security attack (Bhushan \& Sahoo, 2017). There is a literature review focus on Sybil attack countermeasures highlighted by Vasudeva \& Sood (2018), Benkhelifaet et al. (2018) and Gunturu (2015) and its comparison shown in Table 1. A reader interested in Sybil countermeasures in an online network can read the following survey (Al-Qurishi et al., 2017; Alharbi, Zohdy, Debnath, Olawoyin, \& Corser, 2018). However, there is no previous literature that reports cover any Sybil countermeasures based on swarm intelligence to the best of our knowledge. This paper provides a general review of up-to-date countermeasures use to mitigate the Sybil attack. Also, advantages, limitations and whether the existing proposed method is IoT ready are discussed.

The remainder of this paper is organized as follows. The "Survey Methodology" section illustrates the approach and methodology used in this literature review on the Sybil attack. In "Security Attack", we give a general overview of the Sybil attack. Next, we present the existing Sybil attack countermeasures in "Sybil attack countermeasures". In "Discussion", we discuss the comparisons on Sybil countermeasures in WSN and IoT. Section Finally, in "Conclusions", we conclude the survey by summarizing the paper and outlining future research directions.

\section{SURVEY METHODOLOGY}

A systematic literature review ( SLR) was carried out to examine countermeasures suggested by previous research studies to thwart Sybil's attack with the Kitchenham (2004) benchmark, emphasising previous work related to countermeasures for attacks on Sybil. This research approach originated in the medical field to provide adequate knowledge for a repeatable study method (Charband \& Jafari Navimipour, 2016; Jafari Navimipour \& Charband, 2016; Kupiainen, Mäntylä, \& Itkonen, 2015). To guide the reader on why we need to focus on the Sybil attack, to discuss Sybil's critical principles and countermeasures as formalized in the following subsections, we chose four research questions. 


\section{Research Questions}

119 The questions in this section were aimed at identifying the main issues and challenges along with

120

121

122

123

124

125

126

127

128

129

130

131

132

133

134

135

136

137

138

139

140

141

142

143

144

145

146

147

148

149

150

151

152

153

154

155

156

- RQ1: What is Sybil Attack?

- RQ2: Why is it to focus on Sybil IoT-based WSN environment? These two questions will prove the intent of countermeasure for Sybil Attack.

- RQ3: Where will new researchers concentrate on other methods of tackling the attack on Sybil? This problem aims to help the researcher focus on setting the direction of the proposed method.

- RQ4: How can the Sybil countermeasures achieve more robust algorithms to counteract those attacks?

This research question aims to explain how countermeasures are used to thwart Sybil's attack in achieving better algorithms, identifying challenges and techniques. The study needs are established after conducting a search query using suitable keywords, coming up with research questions, identifying the selection criteria, identifying the data retrieval, and conducting the quality evaluation. The aim of the survey could offer prepared answer and enlightenment for new researchers.

\section{Survey Plan and Organization}

The articles in this survey were acquired from most respected academic journals and also selected according to the checklist provided in (Kitchenham et al., 2009; Vasudeva \& Sood, 2018 b) for the quality evaluation. The research articles are acquired included IEEE, Elsevier, Springer, ACM, Wiley and MDPI, as these provided in-depth analysis. We started by filtering article by analysing the titles and abstracts. The entire research article is reviewed when the detailed information was not in the abstract. Hence articles are selected in this analysis based on a detailed inquiry into the nature of their material and documents. This in-depth work enables us to have a consistent and thorough understanding of the countermeasures for Sybil in the IoTbased environment.

The paper analysis was undertaken between late January 2015 and July 2020 for the first filtering. Boolean functions (OR, AND) and specific keywords detailed by synonyms and alternative spellings were used to investigate hundreds of papers in this area further.

("Sybil") and ("attack" or "attacks")

Peer] Comput. Sci. reviewing PDF | (CS-2020:10:54560:1:0:NEW 18 May 2021) 
157

158

159

160

161

162

163

164

165

166

167

168

169

170

171

172

173

174

175

176

177

178

179

180

181

182

183

184

185

186

187

188

189

190

191

192

193

194

195

Next, papers are filtered again to acquire papers more accurately related to the review context. The filtering process is to guarantee that no papers were overlooked in our review using the keywords search below:

("Sybil") and ("attack" or "attacks") and (IoT OR "internet of things") and (WSN OR "wireless sensor networks") -"book" -"conference"

\section{Eligibility Criteria}

Articles were evaluated based on the Quality Assessment Checklist (QAC) to be selected in our survey review list (Kitchenham et al., 2009; Vasudeva \& Sood, 2018b). The articles in this review that matched the research aim and objectives are selected according to the following criteria:

- Does the study paper identify the Sybil attack countermeasure methods?

- Is the methodology listed in the research paper?

- Do testing methodologies use the resources available for re-implementation (simulation or real system)?

- Does the research paper focus on WSN?

- Is the evaluation analysis done appropriately?

If "yes," the papers are chosen after the following conditions have been met:

- Any article that meets the criteria provided when there is a match in the keywords, the article is selected

- The article is filtered after gone through the abstract and later will be recorded in the final list

- Articles related to the countermeasure of the attack on Sybil will be included.

\section{Data Filtration and Quality Evaluation}

The search engine for Google scholars was used to locate the primary studies with the automated search. The search led to the discovery of 28,800 articles that were considered significant for the study. Data for all publications cited, abstracts and keywords of all articles are further analysed in an Excel sheet. Through three articles resulting from the initial search, phase by phase. In this segment, we search for keywords automatically and then find 372 journal articles and conference papers. Then, we include the year range 2010-2020, which is reduced to 333 journals. Then, we choose six famous publishers; 186 articles have been selected. Next, we checked whether any research papers satisfied the criteria or were ignored. When the abstract was found to be inadequate, the entire article was then checked, considering the requirements for inclusion or exclusion given above (Kitchenham et al., 2009). Then, according to the publication time, the number of 28 articles were selected and analysed. Fig. 1 illustrate the procedure used to choose the articles for review. Researchers and scholars mostly published their contributions in the established journal. Hence, conference papers have been excluded from this survey.

Peer] Comput. Sci. reviewing PDF | (CS-2020:10:54560:1:0:NEW 18 May 2021) 
196 Security Attack

197 Generally, the security attack can be classified based on the attacker's objective, on which layer 198 the attack is carried out. The method of attack reviewed in the previous literature is shown in Fig. 199 2. Firstly, objective-based security attack can be divided into the passive and active attack. A 200 passive attack can bring down the network, eavesdropping to collect personal information, node 201 destruction, and node malfunction. In an active attack, the attacker has the objective to take down 202 the targeted network to become useless. Several examples of active attacks can be further 203 classified into flooding, jamming, Denial-of-Service (DoS), black hole, sinkhole, Sybil and 204 wormhole. Secondly, the various passive and active attacks in WSN and IoT can be categorised

205

206

207

208

209

210

211

212

213

214

215

216

217

218

219

220

221

222

223

224

225

226

227

228

229

230

231

232

233 according to OSI layers (Butun, Osterberg, \& Song, 2020; Farjamnia, Gasimov, \& Kazimov, 2019). Different types of attack in the IoT environment are described in (Ahmad \& Salah, 2017). Usman \& Gutierrez ( 2018) categorized the author focus on wormhole attack and as well as other attacks are reviewed in (Farjamnia et al., 2019). Finally, the attack is categorized according to the attack method and how the malicious node can achieve its objective. Besides, the author also highlighted the mitigation strategies against security attacks in Pervasive and Mobile Computing. Sybil, DoS, hello and sinkhole are layered network attacks in WSN that are still relevant in IoT environments (Aufner, 2019). Thus, it is applicable to any IoT devices which uses the communication layer to communicate.

Based on the earlier discussion on the attack, the countermeasures of security attack consist of prevention, detection, and mitigation. Firstly, the prevention method's main objective is to hinder the malicious attack from taking place in the first place. Secondly, the detection countermeasures that able to detect when there is a security breach in the network. The countermeasure method can identify the type of attack and launch the mitigation solution to reduce the damage done by malicious activity, as highlighted in Fig. 3. Hence, the mitigation method is the steps taken to reduce the aftereffect of a security attack. Those three components are a complete protection framework and cannot be considered separately in defence of WSNs and IoT against different types of attacks, as highlighted in (Butun et al., 2020).

Such security attacks cause serious vulnerabilities to be routed inside the underlying network. Many attacks are less extreme, and others more severe (Md Zin, Badrul Anuar, Mat Kiah, \& Ahmedy, 2015). One of the first attacks in the WSN environment is the Sybil attack, leading to further security attack as a black hole and wormhole, as highlighted by Murali \& Jamalipour (2020). These attacks can interrupt the operation of WSN, which considers as IoT devices from collecting data sensors then stored in the cloud. Later, disruption caused IoT application like smart building, which houses many companies, to go haywire. Hence, this review paper focuses on the countermeasure method for Sybil and attack, which will be discussed later in the next section. 


\section{Sybil Attack}

235 Sybil attack is defined by Newsome et al. (2002) when the malicious node can fake its own 236 identity during an attack or stole the identity from working valid nodes. Sybil attack utilises fake 237 identities to send false information, as highlighted by Romadhani et al. (2017) and Zhang et al. 238 (2005). In an ad-hoc network, Sybil attack that utilises multiple fake identities are discussed in 239 Lv et al. (2008). In geographic routing, fake identities exist in the network with faked locations

240

241

242

243

244

245

246

247

248

249

250

251

252

253

254

255

256

257

258

259

260

261

262

263

264

265

266

267

268

269

270

271 explored by Sha et al. (2013) and García-Otero et al. (2010), as shown in Fig. 4. Alternatively, a high-resource Sybil Attack can participate in the selection process by listening and transmitting its fake location during the protocol handshakes. Newsome et al. (2004) highlighted countermeasures for Sybil Attack, namely radio testing and random key pre-distribution. However, the author did not mention any limitation of the sensor network based on the listed method. Goyal et al. (2015) and John et al. (2015) classified the Sybil Attack into a few countermeasures categories.

An attacker tries to get more attention from nearby nodes in this attack to intercept data packets. An attack that affects the process of packet delivery is routed are called routing attacks. The simplest routing attack type is an altering attack in which the attacker modifies the routing information by creating routing loops or fake error messages, as highlighted in Mosenia \& Jha (2017).

WSN security strategies can be broken down into two categories: prevention-based and detection-based. Due to restricted resources and a broadcast medium, mitigation methods such as encryption are challenging for WSNs. Also, all possible attacks will not be protected by the suggested cryptographic solutions. An attacker can easily obtain the symmetric key. The whole network is compromised since the attacker will decrypt all encrypted data using the symmetric key. The second line of defence is called the Intrusion Detection System (IDS), is important to detect malicious parties that try to use the weakness in the security and potential insecurities and detect the attacks that have not been detected before (Hidoussi et al., 2015).

WSN nodes are deployed in the environment without any supervision. The unattended nature of WSNs, adversaries, can readily produce such blackholes attack. Severely compromised node and DOS attacks can interfere with the standard data delivery between sensor nodes and sink or even partition the topology, as Shu et al. (2010) highlighted. In the next chapter, we will address the Sybil Attack countermeasures suggested by the previous researchers.

\section{Sybil Attack Countermeasures}

In this section, the countermeasures used to mitigate the Sybil attack can jeopardize humans' lives by monitoring IoT medical devices or other critical IoT applications. Radio resource testing (RTT) is a countermeasure that can distinguish direct forms of Sybil attack Balachandran \& 
272 Sanyal (2012). Newsome et al. (2004) stated that resource testing is the popular countermeasure

273 to lower the probability of being attack rather than eliminating Sybil attacks for good. Ssu,

274 Wang, \& Chang (2009) proposed the RTT mechanism that assumed nodes in the network could

275 not transmit and receive. Also, Douceur (2007) has proven that a trusted certification method can

276 eliminate the Sybil Attack using a central authority. Some researchers proposed that key

277 management (Paul, Sinha, \& Pal, 2013; Zhang et al., 2005; Eschenauer \& Gligor, 2002) or

278 encryption for authentication using asymmetric key cryptography which is not suitable due to

279 higher overhead and not scalable (Boneh \& Franklin, 2001; Zhu et al. (2006). Ssu et al. (2009).

280 Other methods include Sybil Attacker detecting by verifying neighbouring nodes' set, which

281 caused higher communication overhead. Software-based attestation is a method where the

282 verifier performs through various software or hardware challenges against its neighbouring node

283 (Steiner \& Lupu, 2016).

284

285

286

287

288

289

The radio signal is susceptible to the interference dan signal attenuation caused by the surrounding, which influences the precision of detecting a malicious node using RSSI-based and Time Difference of Arrival-based Scheme(TDOA) based countermeasures. Chan et al. (1994) proposed two localisation methods, namely the estimation of TDOA and solving hyperbolic

290

291

292

293

294

295

296

297

298

299

300

301

302

303

304

305

306

307

308

309

310

311 position. Wen et al. (2008) explained the TDOA ratio with the sender's identity. A Sybil is detected once the beacon nodes calculated and find the same TDOA ratio for two different identities. These countermeasures are no longer in trend as many researchers currently moving towards the proposed method, as shown in Fig. 5. In most current literature, researchers focus on encryption and RSSI mechanisms that $29 \%$ of the solution provided for Sybil countermeasures. The rest of the solutions accounted for $14 \%, 14 \%$ and $7 \%$ for trust, artificial intelligence and encryption hybrid. Lastly, $3 \%$ and $4 \%$ are accounted for rule-based anomaly and multi-kernel.

Li \& Cheffena (2019) proposed a multi-Kernel based expectation-maximization (MKEM) countermeasure for Sybil attacks. The innovative countermeasure analyses the radio resource of the sensor node to produce channel vectors. These channel vectors are comprised of the power gain and delay spread of the channel impulse response extracted out from the received packet of the sensor node. In addition, gap statistical analysis method to validate and EM method to summarize the detection results.

\section{Cryptography}

Cryptography is a popular research area for WSN just before the IoT becomes the technological trend. Although cryptography requires lots of processing power, there is still an ongoing research area among researchers. Kouicem et al. (2018) highlighted two fundamental distribution approaches: key deterministic and Probabilistic key distribution. In deterministic approaches, to provide maximum security coverage connection, each entity can make a secure link to others. However, the key management protocol becomes defenceless when under a security attack. Also, 
312 Paul et al. (2013) highlighted three types of cryptographic technique: creating and managing, 313 distributing, and validating keys for identities. Firstly, Symmetric key distribution is a method 314 using a similar key is utilised for encryption and decryption of the messages, namely Encryption 315 Standard (AES), Rivest Cipher 4(RC4) and Triple Data Encryption Standard (3DES). However, 316 key management problems and scalability issues are the main disadvantages of the symmetric 317 key. WSN nodes are powered by batteries that are not suitable for implementing public-key 318 cryptography due to high processing power and high network load when generating keys, 319 distributing key, and maintaining key. Devices equipped with cryptographic is more likely to expose to brute force attack. Asymmetric key distribution utilised public key for encryption while the private key utilised for decryption.

Jain et al. (2020) proposed a node authentication method for wireless sensor node to avoid security attacks and provide secure communication channels. The base station is responsible for generating the random value and a secret value to distribute among the sensor nodes. Each node is responsible for storing its secret and random value.Zhang \& Zhou (2010) proposed using the Markle hash tree, trust values and message authentication codes for location verification algorithm. This approach works well with networks that are organized in a tree or hierarchical structure. This method falls in the encryption hybrid taxonomy shown in the previous pie chart diagram. Claycomb \& Shin (2011) proposed a method based on a security policy that utilized key establishment to combine group-based distribution model and identitybased encryption. He et al. (2011) proposed combining the merits of both public and symmetric cryptographic methods for key management in WSNs, that each node is configured with a public key system to establish end-to-end symmetric keys with other nodes like EDDK.

Dong \& Liu ( 2012) proposed a scheme that deploys auxiliary nodes to execute the key establishment to help key establishment between sensor nodes. This method utilizes a secured Fuzzy Clustering Algorithm to determine the nodes that securely join the cluster. The cluster head oversees routing based on criteria, trust value and energy. Kim \& Kim (2013) proposed a scalable and robust hierarchical key establishment scheme that enhances resilience against node capture, traffic analysis, and acknowledgement spoofing attack. In addition, this scheme provides periodic critical updates without communication costs for key transport.Razaque \& Rizvi (2017) proposed a method to combat the Sybil attack, which comprises two novel algorithms. The first algorithm fragments the data to avoid detection from the malicious node. The second algorithm aims to provide authentication for nodes joining the network through encryption.

\section{Received Signal Strength Indicator}

Received Signal Strength Indicator (RSSI) has been used to sense Sybil attack and accounted for about 26\% in Fig. 5. RSSI remains the choice of researchers to mitigate against Sybil attack because it does not require special hardware to approximate the location of neighbours. There are 
352

353

354

355

356

357

358

359

360

361

362

363

364

365

366

367

368

369

370

371

372

373

374

375

376

377

378

379

380

381

382

383

384

385

386

387

388

389

390

391

many different approaches in RSSI to counter-attack and which varied between researcher. Demirbas et al. (2006) proposed a countermeasure method Sybil attack by only two receivers. To improve accuracy, Wang et al. (2007) came up with a countermeasure using RSSI from multiple neighbours instead of two neighbour nodes. Also, the status message can be used to validate the location in the hierarchical network that utilises Jake Channel. Zhong et al. (2004) proposed the location verification based on RSSI signal using four or more detector nodes to detect the signals that can verify a node's location.

Lv et al. ( 2008) proposed a method for stationary wireless sensor networks called Cooperative Received Signal Strength (RSS) based Sybil Detection (CRSD) to estimate the distance between two identities and to locate the correlation of location between the unique identities of multiple neighbouring nodes. Lazos et al. (2005) proposed a method that utilises the target node to determine its position using beacon information transmitted by both benevolent and malicious anchor nodes.

García-Otero et al. (2010) proposed innovative and lightweight location verification methods to detect and isolate Sybil attack. The distributed trust model is integrated with the routing protocol mainly to defence from routing attack. Abbas et al. (2013) utilise one neighbouring node to detect RSS in mobile environments. In Secure and Scalable Geographic, Opportunistic Routing with received signal strength (SGOR) is an opportunistic routing protocol proposed by Lyu et al. (2015). This proposed trust method and a combination of calculating the difference of distance beacon messages and RSSI to detect the malicious nodes' fake location and defend against grey hole attack. The proposed method can defend from other attacks such as rushing, wormhole, replay, and collusion. However, this method's limitation is when the attacker has higher energy capacity and higher transmission power, which can easily deceive the sender about its location. Kumari et al. (2017) provided a framework using authentication and RSSI against Sybil attack. The RSS values are calculated from the arrival angle, stored in the database at each node. The RSSI threshold value determines if the nodes fall within the safety zone and the precautionary zone. Also, the ant colony optimisation method was used to determine the optimized route for the packet to travel from source to destination. The second category assumes that a node can occur at one location at a specific time. Raja et al. (2017) suggested another encryption approach using the Fujisaki Okamoto (FO) algorithm and their implementations. FO algorithm is an encryption method that offers a good defence against Sybil attacks by using IDbased verification. In the proposed scheme, multiple performance metrics were analysed, especially the high energy consumption is used as an indicator to sense Sybil attack in wireless sensor networks.

Yuan et al. (2018) presented a lightweight Approximate Point-in Triangulation Test (SFAPIT) algorithm that can pinpoint Sybil attacks in a wireless network in a distributed way using a range of free and iterative refinement-based methods. The individual node implemented the 
392 algorithm was based on RSS, which does not cost any overhead in WSN. Based on the node 393 location, the node utilises three beacons in the triangulation method to calculate the possible 394 combination overlapped triangle region, which can estimate the unknown node's location.

395 Therefore, the centroid of the overlapping area is considered as the approximate location of this

396 node. Giri et al. (2020) proposed a countermeasure that protects the beacon node from Sybil 397 attack by implementing the information-theoretic approach. Any localization algorithm can use 398 this approach to provide protected localization in WSNs for the Sybil attack. Liu (2020)

399

400

401

402

403

404

405

406

407

408

409

410

411

412

413

414

415

416

417

418

419

420

421

422

423

424

425

426

427

428

429

430

431

proposed an improved RSSI-based Sybil Attack Detection Scheme in Wireless Sensor Networks. The proposed method able to quickly detect malicious nodes with minimum energy consumption.

The hierarchical topology of cluster network has many advantages in energy efficiency due to less communication, scalability, and routing. In addition, the proposed method utilised both RSSI and CSI to protect the hierarchical cluster network from Sybil attack. Jamshidi et al. (2019) proposed a lightweight method that consists of two algorithms for detecting Sybil node masquerading as cluster heads and cluster members. Sarigiannidis et al. ( 2015) proposed a secure communication mechanism for clustered WSNs based on the elliptic curve cryptography (ECC) that allows end-users to recover data collected confidentiality. The proposed method has a firm reliance on historical records, making this approach not stable and durable. Angappan et al. (2020) proposed a localized scheme for Sybil node detection called NoSad using RSSI value and the intra-cluster communication, which can be deployed to the device. However, NoSad is not stable when there is a minimum of two Sybil node and cannot cater to mobility in WSN.

Jan et al. (2015) propose an innovative detection countermeasure for Sybil attack in a centralized clustering-based hierarchical network. Sybil nodes with fake identities are detected before the cluster to ensure that usage of the resources is optimized. The detection of Sybil nodes is achieved by analysing the received signal strength from any two high energy nodes. Wang et al. ( 2018) proposed a Sybil attack detection using Channel State Information (CSI) and a self-adaptive multiple signal classification algorithm RSSI for dynamic and static nodes in the clustered network.

\section{Trust}

According to Ishmanov et al. (2017), there is not much research done on security attack detection based on unrelated criteria such as packet drop and packet modification. Mawgoud et al. (2020) highlighted that trust could be set up automatically without personal interaction with previously unregistered and unknown peer neighbours in typical IoT scenarios.

Karlof \& Wagner (2003) highlighted that the trust centre uses a key shared between two nodes for node verification to secure the network. Zhan, Shi, \& Deng ( 2012) proposed the trust management and encryption method that can detect and guess the future behaviour of Sybil

Peer] Comput. Sci. reviewing PDF | (CS-2020:10:54560:1:0:NEW 18 May 2021) 
432 Attacker. During next-hop selection, this trust information is vital to select a safe path to the 433 destination.

434

435

436

437

438

439

440

441

442

443

444

445

446

447

448

449

450

451

452

453

454

455

456

457

458

459

460

461

462

463

464

465

466

467

468

469

470

471

472

473

Zhan et al. ( 2012) proposed selecting the next hop based on trust and energy criteria. The energy watcher module calculates the energy cost for neighbouring nodes and the node's energy, where this information stored in the neighbourhood table. The energy watcher module also approximates the average energy required to route the packet from sender to destination. Alsaedi et al. (2017) proposed a method to detect Sybil attack based on name, location and energy information for each time a new message was routed to the sender. The proposed method also uses a multi-level system where each rule to recognise a Sybil attacker is given to specific agents. These Sybil attackers engage in data aggregation at different stages to collude the aggregated data to disclose invalid data. Also, these malicious nodes may modify and tamper with the timestamps of a message with multiple identities, which can cause havoc to synchronise local clocks in IoT devices. Maddar et al. (2017) proposed an innovative detection method for Sybil nodes with fake identities before the cluster formation in a centralized clustering-based hierarchical network to optimize the usage of the resources. The detection countermeasure works by analyzing neighbouring nodes the received signal strength. Jinhui et al. (2018) proposed a method that can effectively predict energy consumption and increase the detection rate to detect malicious nodes.

Liu et al. (2007) explained that landmarks are required to be trusted. All routing protocols are related to their mechanism of localisation and cannot be isolated from them. Garcia et al. proposed a lightweight method that consists of localisation and intrusion identification techniques using distrusted trust model to thwart several security attacks.Prathusha et al. (2017) proposed secure geographic routing (GSR), which is have been modified from SecuTPGF. GSR's advantage is that it uses low computational power to combat security attacks such as spoofing and an assault on Sybil by introducing SHA-3 node and message authentication. Zhou et al. (2015) proposed a watchdog method that implements energy consumption optimisation while providing just enough security. The validation technique through a watchdog mechanism able to defend against Sybil attack.

\section{Artificial Intelligent}

Intrusion detection systems are the example of artificial intelligence applications in the cybersecurity field. Cybersecurity solutions can distinguish between legitimate or malicious node through detailed traffic analysis Cyberattacks were first detected with rule-based systems, which could detect attacks based on their signatures at the beginning of the Internet. Swarm Intelligence (SI) is a subdivision of artificial intelligence where the inspiration of this algorithm mimics biological swarms' intelligent behaviour in solving and simulating real problems. The SI algorithms are intended to investigate the concepts of simple individuals who can display sophisticated and complex swarm optimization behaviours through collaboration, organisation,

Peer] Comput. Sci. reviewing PDF | (CS-2020:10:54560:1:0:NEW 18 May 2021) 
474 knowledge exchange, and learning between swarm members.(Kolias, Kambourakis, \& 475 Maragoudakis, 2011). These swarm intelligences can be categorized according to the year when 476 they are invented. Particle Swarm Optimization and Ant Colony Optimization was invented before 477 the year 2000. Artificial Fish Swarm and Bacterial Foraging Optimization need further 478 development to enhance, and Firefly Optimization and Artificial Bee Colony optimization are 479 widely used optimization during the year 2000 until 2010. Pigeon inspired optimization, Grey wolf 480 481 optimizer, and Butterfly optimization algorithm require further development.

Prithi \& Sumathi (2020) proposed a method called Learning Dynamic Deterministic Finite Automata (LD² FA) and Particle Swarm Optimization (PSO) for intrusion detection, and the data is transmitted securely over-optimized path. LD2FA-PSO got a $16 \%$ increase in throughput than cluster-based IDS, almost 70\% rise in throughput than lightweight IDS, $6 \%$ and $32 \%$ increment in network lifetime over PSO and GLBCA, respectively; almost 30\% and 54\% improve in network

487

488

489

490

491

492

493

494

495

496

497

498

499

500

501

502

503

504

505

506

507

508

509

510

511

512

513

514 lifetime over GA and LDC, respectively. The energy consumed is almost $3 \%$ and $6 \%$ lesser than PSO and GA, and 13\% higher energy is consumed than LDC.

Raghav et al. (2020) used swarm intelligence algorithms based on the bee to provide a secure routing scheme. The proposed routing mechanism utilize primary scout bee and secondary scout bee to carried out the secured and optimized routing. In many scenarios, it improves data efficiency while also providing security against flood, spoof, and Sybil attacks. Its disadvantages include that when the solution is close to the global optimum, it is possible to get stuck in the local optimum, resulting in stagnation.

\section{Discussion}

This paper has reviewed the countermeasures used to defence against Sybil attack. Table 3 provides a comparative summary of the proposed method to countermeasure against Sybil attack in term of its advantage, limitation, scalability readiness and classification of detection, prevention. Besides security, scalability is also essential for deploying many devices under the IoT paradigm to become a major success (Arellanes \& Lau, 2020). Security countermeasures should expand to many sensor nodes and intelligent devices ( $\mathrm{Lu} \& \mathrm{Xu}, 2019$ ). Comparing the proposed method will help the future researcher evaluate and identify any research gap that will help them innovate or develop new countermeasures in the future. The proposed method to combat Sybil attack is the random key pre-distribution, cryptographic method, radio resource testing, received signal strength indicator (RSSI) localisation techniques, time difference of arrival (TDOA) localisation technique, neighbouring node information, Trust, watchdog, RFID, clustering, and geographic routing.

Sybil attack countermeasures are of the simplified method due to the neighbouring node, and trust information is exchanges of control message between one or more nodes so that the sender can validate the identity of its neighbouring nodes. Also, this information is used as 
515 criteria's in selecting the best route from the sender to the destination nodes. Watchdog is used to 516 monitor the neighbouring nodes in a centralised or decentralised scheme using the physical and

517 data link layer. This information is used in selecting the best route for multi-hop routing.

518

519

520

521

522

523

524

525

526

527

528

529

530

531

532

533

534

535

536

537

538

539

540

541

542

543

544

545

546

547

548

549

550

551

552

553

554
Cryptographic and random key pre-distribution is implemented in the application layer, where its encryption and decryption process utilises the processing and memory resources. However, this authentication using asymmetric key cryptography has a higher overhead and not scalable. Also, the encryption process requires high computation and memory resources for the cryptography method and its attributes. The limitation for pre-distribution of the key. However, the proposed method utilises high computational overhead, computational delays and a high load of control messages transmitted to nodes. Keys are store in databases that are vulnerable to attacks. One of the significant challenges is developing a lightweight key delivery network for sensor nodes with limited resources to support numerous protocols, applications and services at all IoT layers levels (B. B. Gupta \& Quamara, 2018).

Radio resource testing, RSSI and TDOA measure the physical layers described by Almas Shehni et al. (2017) for the Sybil attack. RSSI and TDOA are two methods to locate Sybil Attack by measuring signal strength and the distance between beacons. The RSSI method used less energy than other methods and did not require any special requirements or additional details. According to the studies, the distances between nodes from the RSSI can be easily calculated based on RSSI information. RSSI-countermeasure methods are popular among researchers to detect Sybil Attack (Demirbas \& Song, 2006). However, the limitation of RSSI are susceptible to interference, environment factor, the need for a beacon node, receiver system delay, non-line of sight transmission, and a malicious node with high power transmission could easily deceive the good node with its fake location and identity. The disadvantage of TDOA is implemented in a highly dense network which can cause false detection of an honest node being detected as an attacker. An honest node's location is at the exact location as the detector node are the leading cause of false detection. Also, an attacker with a directional antenna could easily overcome being detected. These methods are not suitable for IoT devices that are mobile (Wu \& Ma, 2019). RSSI has some limitation where there is no line of sight communication due to the obstruction of obstacle between a beacon node and a dumb node which caused the signal to get reflected from the surroundings (Ren et al., 2007). Hence, from the summary of countermeasures proposed by the previous researcher, the future researcher should use RSSI due to its energy efficiency. To complement the limitation of RSSI, trust countermeasures based on energy due to energy heterogeneity of IoT devices should be combined with RSSI to enable the detection of Sybil malicious nodes.

Software attestation is a method where software routines that transmitted to the neighbouring node for validation. These routines are stored inside the memory, and the neighbouring nodes are required to respond to the challenge within specific criteria like integrity

Peer] Comput. Sci. reviewing PDF | (CS-2020:10:54560:1:0:NEW 18 May 2021) 
555

556

557

558

559

560

561

562

563

564

565

566

567

568

569

570

571

572

573

574

575

576

577

578

579

580

581

582

583

584

585

586

587

588

589

590

591

592

593

594

595

validation in software and hardware, time duration, how software routine is read in the memory, and the interaction method. For example, the radio resource testing technique extracts the battery or energy level from these network devices. High energy devices are assumed to be malicious attacker nodes. However, this approach can cause the communication overhead to increase due to control packets for resource verification.

Fig. 6 illustrates the proposed method that the researcher has developed from the year 2010 until 2020. The statistical charts show an increase in the encryption and trust method proposed by the researchers in 2017. In the year 2020, there is an increase in the proposed method using RSSI and less focus on encryption. Most of the artificial intelligence scheme proposed by the researchers in 2020 is used to optimize the routing process in complement with security. Hence, in the next section future researcher should try to integrate artificial intelligence to optimize the method such as cross layer, Software Defined Network (SDN), cross platform intrusion detection and blockchain.

\section{Lesson learned and future direction}

WSN security is a hot research topic. There are many challenges and issues in WSN's security which future researcher can explore and provide a new solution. Specific requirements and constraints, such as low complexity and reliability, must be imposed on the provided solution. This section briefly discusses lessons learned from the previously proposed method and possible future directions for Sybil Attack countermeasures.

\section{Cross-layer}

Lesson learned: There is a possibility that an attack could be launch from the different layer during the communication process. Hence, this requires security countermeasure to handle cross-layer attacks and require access to all information from multiple layers. Significantly through joint optimization of multiple network layers. Besides security, cross-layer information also beneficial in term of optimizing energy efficiency

Dhivya Devi \& Vidya (2019) discussed and explored the cross-layer design approaches that have been in WSN. For example, some proposed methods implement a cross-layer in detecting intrusion and routing (Fatema \& Brad, 2013; Umar et al., 2017). The motivation to implement cross-layer design due to it can optimise the network performance in the wireless sensor. The cross-layer design allows the ease of exchanging information between layers, which helps the WSN be energy efficient and increase QoS parameters. Based on the proposed method for Sybil attack, countermeasures surveyed, not many works of literature in focusing security attack. The method in detecting Sybil attack should incorporate the cross-layer approach to increase accuracy in detecting security attacks. The future researcher can utilise the RSSI, which 
596 lightweight from the physical layer with an upper layer such as Trust and Mobile agent for 597 detecting Sybil Attack. A cross-layer method for detecting Sybil attack with a mobile agent is 598 proposed by Gandhimathi (2016). Cross-layer allows sharing of information among the MAC 599 and networks layers is to optimise network performance. Also, this information can utilise by a 600 mobile agent to prevent a security attack. However, the proposed method to prevent Sybil attack 601 and another kind of attack increases the communication overhead.

602

603 Software-Defined Network

604

605

Lessons learned: SDN and SDMN are the current trending research topics for 5G communication

606 security. The exact security method for SDN and SDMN remain unexplored by researchers.

607 With the deployment of SDN and SDMN in the 5G communication, innovative techniques are

608 needed in this area.

609

610

Apart from novel security solutions for IoT, there is a developing trend of SDN that allows reconfiguration of the network and central monitoring with possible centralised routing 612 algorithm. This emerging paradigm opens up the researcher's door to develop a lightweight 613 security framework running from the SDN controller, running at the central controller (Hameed, 614 Khan, \& Hameed, 2019).

615

616

\section{Cross-Platform Intrusion Detection}

617

618

619

Lesson learned: The IoT has from WSN where the sensor nodes are assumed to homogenous device with limited resources to heterogeneous devices with different capabilities but still limited

620 in energy constraints. Colom et al. (2018) highlighted in the survey that the current trend of IDS is

621

622 moving toward a universal and cross-platform method. The proposed method able to handle device heterogeneity, scalability of IoT network and limitation.

623

624

625

626

627

628

629

630

631

632

633

634

Security and malware attack on the Internet could also be deployed in IoT due to various protocols utilized at every layer in the heterogeneous devices. The interoperability issues and lack of standard in IoT becomes a security challenge. Many IoT devices that launched in the market have a security flaw due to that security was not the top priority and have not been considered in the past. The previous IoT devices are lack authentication method or able to detect or prevent an attack. A big challenge for Intrusion detection methods to be deployed in the IoT environment is a big challenge due to the heterogeneity of devices. One example cross-platform intrusion detection; an innovative home application must retrieve information from personal healthcare sensing with a secure connection. Therefore, we need a quick, efficient and robust intrusion detection countermeasure to provide an undisruptive and continuous connection to multiple IoT platforms.

635 
636

637

638

639

640

641

642

643

644

645

646

647

648

649

650

651

652

653

654

655

656

657

658

659

660

661

662

663

664

665

666

667

668

669

670

671

672

673

674

675

676

\section{Blockchain}

Lesson learned: Blockchain is the latest decentralised distributed system technology designed and invented by Bayer et al. in 1992. Proof of work, asymmetric cryptography, electronic signatures, and hash functions are all used in blockchain technology (Lazrag, Chehri, Saadane, \& Rahmani, 2020).

WSN sensor nodes are distributed and placed in an extreme and complex environment, so it is crucial to implement secure authentication between sensor nodes in WSN(Cui et al., 2020). Blockchain is suitable for IoT with a hierarchical topology that has limited memory, computation, and energy. Merkle trees were incorporated into blockchain technology to provide efficient and reliable digital timestamps (Dorri et al., 2017). Blockchain has been applied in the security framework, and security countermeasure is still in the experimental phase, which will be the future direction of the research (Mubarakali, 2021)

\section{Conclusions}

This paper discussed different countermeasures to defend the IoT-based WSN from the Sybil attack launched from various application domains. We have expanded on their modus operandi, advantages, and limitations of each countermeasure's categories. Although various researchers have proposed several countermeasures, there is no efficient method to overcome most attacks with complete geographic routing accuracy. Also, we have observed that the trust mechanism is the most popular countermeasures for the Sybil attack from 2015 and 2020.

The new researcher should investigate developing a framework that is lightweight to secure IoT network. Developing a secure framework for IoT, which consists of heterogeneous devices with different wireless technologies, is challenging. The development of a security framework for these IoT devices should consider IoT's scalability and resource constraint (Razacheema, Alsmadi, \& Ikki, 2018).

\section{Acknowledgements}

This work was supported by Geran Putra Berimpak Universiti Putra Malaysia (9659400).

\section{References}

Abbas, S., Merabti, M., Llewellyn-Jones, D., \& Kifayat, K. (2013). Lightweight sybil attack detection in MANETs. IEEE Systems Journal, 7(2), 236-248. https://doi.org/10.1109/JSYST.2012.2221912

Ahmad, M., \& Salah, K. (2017). IoT security : Review , blockchain solutions, and open challenges. Future Generation Computer Systems. https://doi.org/10.1016/j.future.2017.11.022

Al-Qurishi, M., Al-Rakhami, M., Alamri, A., AlRubaian, M., Rahman, S. M. M., \& Hossain, M. 
677

678

679

680

681

682

683

684

685

686

687

688

689

690

691

692

693

694

695

696

697

698

699

700

701

702

703

704

705

706

707

708

709

710

711

712

713

714

715

716

717

718

719

720

721

722
S. (2017). Sybil defense techniques in online social networks: A survey. IEEE Access, 5, 1200-1219. https://doi.org/10.1109/ACCESS.2017.2656635

Ala'Anzy, M., \& Othman, M. (2019). Load Balancing and Server Consolidation in Cloud Computing Environments: A Meta-Study. IEEE Access, 7, 141868-141887. https://doi.org/10.1109/access.2019.2944420

Alharbi, A., Zohdy, M., Debnath, D., Olawoyin, R., \& Corser, G. (2018). Sybil Attacks and Defenses in Internet of Things and Mobile Social Networks. International Journal of Computer Science Issues, 15(6), 36-41.

Almas Shehni, R., Faez, K., Eshghi, F., \& Kelarestaghi, M. (2017). A New Lightweight Watchdog-Based Algorithm for Detecting Sybil Nodes in Mobile WSNs. Future Internet, 10(1), 1. https://doi.org/10.3390/fi10010001

Ambarkar, S. S., \& Shekokar, N. (2020). Toward Smart and Secure IoT Based Healthcare System (Vol. 266, pp. 283-303). Springer International Publishing. https://doi.org/10.1007/978-3-030-39047-1_13

Angappan, A., Saravanabava, T. P., Sakthivel, P., \& Vishvaksenan, K. S. (2020). Novel Sybil attack detection using RSSI and neighbour information to ensure secure communication in WSN. Journal of Ambient Intelligence and Humanized Computing, (0123456789). https://doi.org/10.1007/s12652-020-02276-5

Arellanes, D., \& Lau, K. K. (2020). Evaluating IoT service composition mechanisms for the scalability of IoT systems. Future Generation Computer Systems, 108, 827-848. https://doi.org/10.1016/j.future.2020.02.073

Atzori, L., Iera, A., \& Morabito, G. (2010). The Internet of Things: A survey. Computer Networks, 54(15), 2787-2805. https://doi.org/10.1016/j.comnet.2010.05.010

Aufner, P. (2019). The IoT security gap: a look down into the valley between threat models and their implementation. International Journal of Information Security. https://doi.org/10.1007/s10207-019-00445-y

Balachandran, N., \& Sanyal, S. (2012). A Review of Techniques to Mitigate Sybil Attacks. International Journal of Advanced Networking and Applications, 4, 1-6.

Behera, T. M., Mohapatra, S. K., Samal, U. C., Khan, M. S., Daneshmand, M., \& Gandomi, A. H. (2020). I-SEP: An Improved Routing Protocol for Heterogeneous WSN for IoT-Based Environmental Monitoring. IEEE Internet of Things Journal, 7(1), 710-717. https://doi.org/10.1109/JIOT.2019.2940988

Benkhelifa, E., Welsh, T., \& Hamouda, W. (2018). A critical review of practices and challenges in intrusion detection systems for IoT: Toward universal and resilient systems. IEEE Communications Surveys and Tutorials, 20(4), 3496-3509. https://doi.org/10.1109/COMST.2018.2844742

Bhushan, B., \& Sahoo, G. (2017). Recent Advances in Attacks , Technical Challenges, Vulnerabilities and Their Countermeasures in Wireless. Wireless Personal Communications. https://doi.org/10.1007/s11277-017-4962-0

Boneh, D., \& Franklin, M. (2001). Identity-Based Encryption from the Weil Pairing. In Advances in the Astronautical Sciences (Vol. 102 I, pp. 213-229). https://doi.org/10.1007/3540-44647-8_13

Butun, I., Osterberg, P., \& Song, H. (2020). Security of the Internet of Things: Vulnerabilities, Attacks, and Countermeasures. IEEE Communications Surveys and Tutorials, 22(1), 616644. https://doi.org/10.1109/COMST.2019.2953364

Chan, Y. T., \& Ho, K. C. (1994). A simple and efficient estimator for hyperbolic location. IEEE 
723

724

725

726

727

728

729

730

731

732

733

734

735

736

737

738

739

740

741

742

743

744

745

746

747

748

749

750

751

752

753

754

755

756

757

758

759

760

761

762

763

764

765

766

767

768
Transactions on Signal Processing, 42(8), 1905-1915. https://doi.org/10.1109/78.301830

Claycomb, W. R., \& Shin, D. (2011). A novel node level security policy framework for wireless sensor networks. Journal of Network and Computer Applications, 34(1), 418-428. https://doi.org/10.1016/j.jnca.2010.03.004

Colom, J. F., Gil, D., Mora, H., Volckaert, B., \& Jimeno, A. M. (2018). Scheduling framework for distributed intrusion detection systems over heterogeneous network architectures. Journal of Network and Computer Applications, 108, 76-86. https://doi.org/10.1016/j.jnca.2018.02.004

Cui, Z., Xue, F., Zhang, S., Cai, X., Cao, Y., Zhang, W., \& Chen, J. (2020). A Hybrid BlockChain-Based Identity Authentication Scheme for Multi-WSN. IEEE Transactions on Services Computing, 13(2), 241-251. https://doi.org/10.1109/TSC.2020.2964537

Demirbas, M., \& Song, Y. (2006). An RSSI-based scheme for sybil attack detection in wireless sensor networks. Proceedings - WoWMoM 2006: 2006 International Symposium on a World of Wireless, Mobile and Multimedia Networks, 2006, 564-568. https://doi.org/10.1109/WOWMOM.2006.27

Dhivya Devi, C., \& Vidya, K. (2019). A Survey on Cross-Layer Design Approach for Secure Wireless Sensor Networks. In International Conference on Innovative Computing and Communications (Vol. 55, pp. 43-59). Springer Singapore. https://doi.org/10.1007/978981-13-2324-9_6

Dong, Q., \& Liu, D. (2012). Using auxiliary sensors for pairwise key establishment in WSN. Transactions on Embedded Computing Systems, 11(3). https://doi.org/10.1145/2345770.2345771

Douceur, J. R. (2007). The Sybil Attack, 251-260. https://doi.org/10.1007/3-540-45748-8_24

El-Rashidy, N., El-Sappagh, S., Islam, S. M. R., El-Bakry, H. M., \& Abdelrazek, S. (2020). EndTo-End Deep Learning Framework for Coronavirus (COVID-19) Detection and Monitoring. Electronics, 9(9), 1439. https://doi.org/10.3390/electronics9091439

Eschenauer, L., \& Gligor, V. D. (2002). A key-management scheme for distributed sensor networks. Proceedings of the 9th ACM Conference on Computer and Communications Security - CCS '02, 41. https://doi.org/10.1145/586115.586117

Farjamnia, G., Gasimov, Y., \& Kazimov, C. (2019). Review of the Techniques Against the Wormhole Attacks on Wireless Sensor Networks. Wireless Personal Communications, 105(4), 1561-1584. https://doi.org/10.1007/s11277-019-06160-0

Fatema, N., \& Brad, R. (2013). Attacks and Counterattacks on Wireless Sensor Networks. International Journal of Ad Hoc, Sensor \& Ubiquitous Computing, 4(6), 1-15. https://doi.org/10.5121/ijasuc.2013.4601

Fedele, R., \& Merenda, M. (2020). An IoT System for Social Distancing and Emergency Management in Smart Cities Using Multi-Sensor Data, 1-24.

Gandhimathi, L., \& Murugaboopathi, G. (2016). Cross layer intrusion detection and prevention of multiple attacks in Wireless Sensor Network using Mobile agent. In 2016 International Conference on Information Communication and Embedded Systems, ICICES 2016 (pp. 15). IEEE. https://doi.org/10.1109/ICICES.2016.7518935

García-Otero, M., Zahariadis, T., Álvarez, F., Leligou, H., Población-Hernández, A., Karkazis, P., \& Casajús-Quirós, F. (2010). Secure Geographic Routing in Ad Hoc and Wireless Sensor Networks. EURASIP Journal on Wireless Communications and Networking, 2010(1), 975607. https://doi.org/10.1155/2010/975607

Giri, A., Dutta, S., \& Neogy, S. (2020). Information-theoretic approach for secure localization 
769

770

771

772

773

774

775

776

777

778

779

780

781

782

783

784

785

786

787

788

789

790

791

792

793

794

795

796

797

798

799

800

801

802

803

804

805

806

807

808

809

810

811

812

813

814 against sybil attack in wireless sensor network. Journal of Ambient Intelligence and Humanized Computing, (0123456789). https://doi.org/10.1007/s12652-020-02690-9

Goyal, S. (2015). Wormhole and Sybil Attack in WSN : A Review. 2015 2nd International Conference on Computing for Sustainable Global Development (INDIACom), 1463-1468. Retrieved from https://ieeexplore.ieee.org/abstract/document/7100491/

Gunturu, R. (2015). Survey of Sybil Attacks in Social Networks. Scandinavian Journal of Surgery, 98(1), 25-29. https://doi.org/10.1177/145749690909800105

Gupta, B. B., \& Quamara, M. (2018). An overview of Internet of Things (IoT): Architectural aspects, challenges, and protocols. Concurrency and Computation: Practice and Experience, (June), e4946. https://doi.org/10.1002/cpe.4946

Gupta, D., Bhatt, S., Gupta, M., \& Tosun, A. S. (2020). Future Smart Connected Communities to Fight COVID-19 Outbreak, 1-35. Retrieved from http://arxiv.org/abs/2007.10477

Hameed, S., Khan, F. I., \& Hameed, B. (2019). Understanding Security Requirements and Challenges in Internet of Things (IoT): A Review. Journal of Computer Networks and Communications, 2019. https://doi.org/10.1155/2019/9629381

Ishmanov, F., \& Bin Zikria, Y. (2017). Trust Mechanisms to Secure Routing in Wireless Sensor Networks: Current State of the Research and Open Research Issues. Journal of Sensors, 2017, 1-16. https://doi.org/10.1155/2017/4724852

Jain, U., Hussain, M., \& Kakarla, J. (2020). Simple, secure, and lightweight mechanism for mutual authentication of nodes in tiny wireless sensor networks. International Journal of Communication Systems, 33(9), 1-16. https://doi.org/10.1002/dac.4384

Jamshidi, M., Zangeneh, E., Esnaashari, M., Darwesh, A. M., \& Meybodi, M. R. (2019). A Novel Model of Sybil Attack in Cluster-Based Wireless Sensor Networks and Propose a Distributed Algorithm to Defend It. Wireless Personal Communications, 105(1), 145-173. https://doi.org/10.1007/s11277-018-6107-5

Jan, M. A., Nanda, P., He, X., \& Liu, R. P. (2015). A sybil attack detection scheme for a centralized clustering-based hierarchical network. Proceedings - 14th IEEE International Conference on Trust, Security and Privacy in Computing and Communications, TrustCom 2015, 1, 318-325. https://doi.org/10.1109/Trustcom.2015.390

Jawad, H. M., Nordin, R., \& Gharghan, S. K. (2017). Energy-Efficient Wireless Sensor Networks for Precision Agriculture : A Review. https://doi.org/10.3390/s17081781

Jinhui, X., Yang, T., Feiyue, Y., Leina, P., Juan, X., \& Yao, H. (2018). Intrusion detection system for hybrid DoS attacks using energy trust in wireless sensor networks. Procedia Computer Science, 131, 1188-1195. https://doi.org/10.1016/j.procs.2018.04.297

John, R., Cherian, J. P., \& Kizhakkethottam, J. J. (2015). A survey of techniques to prevent sybil attacks. Proceedings of the IEEE International Conference on Soft-Computing and Network Security, ICSNS 2015, 1-6. https://doi.org/10.1109/ICSNS.2015.7292385

Karlof, C., \& Wagner, D. (2003). Secure routing in wireless sensor networks: attacks and countermeasures. Proceedings of the First IEEE International Workshop on Sensor Network Protocols and Applications, 2003., 113-127. https://doi.org/10.1109/SNPA.2003.1203362

Khanna, A., \& Tomar, R. (2016). IoT based Interactive Shopping Ecosystem. 2016 2nd International Conference on Next Generation Computing Technologies (NGCT), (October), 40-45. https://doi.org/10.1109/NGCT.2016.7877387

Kim, J., \& Kim, K. (2013). A scalable and robust hierarchical key establishment for missioncritical applications over sensor networks. Telecommunication Systems, 52(2), 1377-1388.

Peer] Comput. Sci. reviewing PDF | (CS-2020:10:54560:1:0:NEW 18 May 2021) 
815

816

817

818

819

820

821

822

823

824

825

826

827

828

829

830

831

832

833

834

835

836

837

838

839

840

841

842

843

844

845

846

847

848

849

850

851

852

853

854

855

856

857

858

859

860

https://doi.org/10.1007/s11235-011-9650-x

Kitchenham, B., Pearl Brereton, O., Budgen, D., Turner, M., Bailey, J., \& Linkman, S. (2009). Systematic literature reviews in software engineering - A systematic literature review. Information and Software Technology, 51(1), 7-15. https://doi.org/10.1016/j.infsof.2008.09.009

Kolias, C., Kambourakis, G., \& Maragoudakis, M. (2011). Swarm intelligence in intrusion detection: A survey. Computers and Security, 30(8), 625-642. https://doi.org/10.1016/j.cose.2011.08.009

Kouicem, D. E., Bouabdallah, A., \& Lakhlef, H. (2018). Internet of things security: A top-down survey. Computer Networks, 141, 199-221. https://doi.org/10.1016/j.comnet.2018.03.012

Kumari, P., \& Sahana, S. K. (2019). An Efficient Swarm-Based Multicast Routing TechniqueReview. In H. S. Behera, J. Nayak, B. Naik, \& A. Abraham (Eds.) (Vol. 711, pp. 123-134). Singapore: Springer Singapore. https://doi.org/10.1007/978-981-10-8055-5_12

Lazos, L., \& Poovendran, R. (2005). SeRLoc: Robust Localization for Wireless Sensor Networks. ACM Transactions on Sensor Networks, 1(1), 73-100. https://doi.org/10.1145/1077391.1077395

Lazrag, H., Chehri, A., Saadane, R., \& Rahmani, M. D. (2020). Efficient and secure routing protocol based on Blockchain approach for wireless sensor networks. Concurrency Computation , (November), 1-10. https://doi.org/10.1002/cpe.6144

Li, Q., \& Cheffena, M. (2019). Exploiting Dispersive Power Gain and Delay Spread for Sybil Detection in Industrial WSNs: A Multi-Kernel Approach. IEEE Transactions on Wireless Communications, 18(3), 1805-1818. https://doi.org/10.1109/TWC.2019.2897308

Liu, G., Wang, X., Li, X., Hao, J., \& Feng, Z. (2018). ESRQ: An Efficient Secure Routing Method in Wireless Sensor Networks Based on Q-Learning. Proceedings - 17th IEEE International Conference on Trust, Security and Privacy in Computing and Communications and 12th IEEE International Conference on Big Data Science and Engineering, Trustcom/BigDataSE 2018, 149-155. https://doi.org/10.1109/TrustCom/BigDataSE.2018.00032

Liu, K., Abu-Ghazaleh, N., \& Kang, K. D. (2007). Location verification and trust management for resilient geographic routing. Journal of Parallel and Distributed Computing, 67(2), 215-228. https://doi.org/10.1016/j.jpdc.2006.08.001

Lu, Y., \& Xu, L. Da. (2019). Internet of things (IoT) cybersecurity research: A review of current research topics. IEEE Internet of Things Journal, 6(2), 2103-2115. https://doi.org/10.1109/JIOT.2018.2869847

Luo, Y. (2019). Data Collection Through Mobile Vehicles in Edge Network of Smart City. IEEE Access, 7, 168467-168483. https://doi.org/10.1109/ACCESS.2019.2951587

Lv, S., Wang, X., Zhao, X., \& Zhou, X. (2008). Detecting the Sybil attack cooperatively in wireless sensor networks. Proceedings - 2008 International Conference on Computational Intelligence and Security, CIS 2008, 1, 442-446. https://doi.org/10.1109/CIS.2008.69

Lyu, C., Gu, D., Zhang, X., Sun, S., Zhang, Y., \& Pande, A. (2015). SGOR: Secure and scalable geographic opportunistic routing with received signal strength in WSNs. Computer Communications, 59, 37-51. https://doi.org/10.1016/j.comcom.2015.01.003

Maddar, H., Kammoun, W., \& Youssef, H. (2017). Trust Intrusion Detection System Based on Location for Wireless Sensor Network. In A. M. Madureira, A. Abraham, D. Gamboa, \& P. Novais (Eds.) (Vol. 557, pp. 831-840). Cham: Springer International Publishing. https://doi.org/10.1007/978-3-319-53480-0_82

Peer) Comput. Sci. reviewing PDF | (CS-2020:10:54560:1:0:NEW 18 May 2021) 
861

862

863

864

865

866

867

868

869

870

871

872

873

874

875

876

877

878

879

880

881

882

883

884

885

886

887

888

889

890

891

892

893

894

895

896

897

898

899

900

901

902

903

904

905

906

Mawgoud, A. A., Taha, M. H. N., \& Khalifa, N. E. M. (2020). Security Threats of Social Internet of Things in the Higher Education Environment (Vol. 846, pp. 151-171). Springer International Publishing. https://doi.org/10.1007/978-3-030-24513-9_9

Md Zin, S., Badrul Anuar, N., Mat Kiah, M. L. M., \& Ahmedy, I. (2015). Survey of secure multipath routing protocols for WSNs. Journal of Network and Computer Applications, 55, 123-153. https://doi.org/10.1016/j.jnca.2015.04.018

Mosenia, A., \& Jha, N. K. (2017). A comprehensive study of security of internet-of-things. IEEE Transactions on Emerging Topics in Computing, 5(4), 586-602. https://doi.org/10.1109/TETC.2016.2606384

Mubarakali, A. (2021). An Efficient Authentication Scheme Using Blockchain Technology for Wireless Sensor Networks. Wireless Personal Communications, (0123456789). https://doi.org/10.1007/s11277-021-08212-w

Murali, S., \& Jamalipour, A. (2020). A Lightweight Intrusion Detection for Sybil Attack under Mobile RPL in the Internet of Things. IEEE Internet of Things Journal, 7(1), 379-388. https://doi.org/10.1109/JIOT.2019.2948149

Newsome, J., Shi, E., Song, D., \& Perrig, A. (2004). The sybil attack in sensor networks, 259. https://doi.org/10.1145/984622.984660

Paul, A., Sinha, S., \& Pal, S. (2013). An Efficient Method to Detect Sybil Attack using Trust based Model. Proc. of Int. Conf. on Advances in Computer Science, AETACS An.

Prathusha Laxmi, B., \& Chilambuchelvan, A. (2017). GSR: Geographic Secured Routing using SHA-3 algorithm for node and message authentication in wireless sensor networks. Future Generation Computer Systems, 76, 98-105. https://doi.org/10.1016/j.future.2017.05.015

Prithi, S., \& Sumathi, S. (2020). LD2FA-PSO: A novel Learning Dynamic Deterministic Finite Automata with PSO algorithm for secured energy efficient routing in Wireless Sensor Network. Ad Hoc Networks, 97, 102024. https://doi.org/10.1016/j.adhoc.2019.102024

Pundir, S., Wazid, M., \& Singh, D. P. (2020). Intrusion Detection Protocols in Wireless Sensor Networks Integrated to Internet of Things Deployment : Survey and Future Challenges. IEEE Access, 8, 3343-3363. https://doi.org/10.1109/ACCESS.2019.2962829

Qinghua Zhang, Pan Wang, Reeves, D. S., \& Peng Ning. (2005). Defending against Sybil Attacks in Sensor Networks, 185-191. https://doi.org/10.1109/icdesw.2005.57

Razacheema, A., Alsmadi, M., \& Ikki, S. (2018). Survey of Identity-Based Attacks Detection Techniques in Wireless Networks Using Received Signal Strength. Canadian Conference on Electrical and Computer Engineering, 2018-May. https://doi.org/10.1109/CCECE.2018.8447756

Razaque, A., \& Rizvi, S. S. (2017). Secure Data Aggregation Using Access Control and Authentication for Wireless Sensor Networks. Computers \& Security. https://doi.org/10.1016/j.cose.2017.07.001

Ren, K., Lou, W., Zeng, K., Member, S., Moran, P. J., Sohraby, K., ... Znati, T. (2007). Wireless Sensor Networks. Booksgooglecom (Vol. 6). Retrieved from https://dl.acm.org/citation.cfm?id=2480912

Roy Chowdhury, A. (2017). IoT and Robotics: a synergy. PeerJ, 5. https://doi.org/10.7287/peerj.preprints. 2760

Santos, C., Jimenez, J. A., \& Espinosa, F. (2019). Effect of Event-Based Sensing on IoT Node Power Efficiency. Case Study: Air Quality Monitoring in Smart Cities. IEEE Access, 7, 132577-132586. https://doi.org/10.1109/ACCESS.2019.2941371

Sha, K., Gehlot, J., \& Greve, R. (2013). Multipath routing techniques in wireless sensor

Peer] Comput. Sci. reviewing PDF | (CS-2020:10:54560:1:0:NEW 18 May 2021) 
907

908

909

910

911

912

913

914

915

916

917

918

919

920

921

922

923

924

925

926

927

928

929

930

931

932

933

934

935

936

937

938

939

940

941

942

943

944

945

946

947

948

949

950

951

952

networks: A survey. Wireless Personal Communications, 70(2), 807-829.

https://doi.org/10.1007/s11277-012-0723-2

Sheron, P. S. F., Sridhar, K. P., Baskar, S., \& Shakeel, P. M. (2020). A decentralized scalable security framework for end-to-end authentication of future IoT communication.

Transactions on Emerging Telecommunications Technologies, 31(12), 1-12. https://doi.org/10.1002/ett.3815

Sikder, A. K., Petracca, G., Aksu, H., Jaeger, T., \& Uluagac, A. S. (2018). A Survey on Sensorbased Threats to Internet-of-Things (IoT) Devices and Applications. Retrieved from http://arxiv.org/abs/1802.02041

Ssu, K. F., Wang, W. T., \& Chang, W. C. (2009). Detecting Sybil attacks in Wireless SeSsu, K. F., Wang, W. T., \& Chang, W. C. (2009). Detecting Sybil attacks in Wireless Sensor Networks using neighboring information. Computer Networks, 53(18), 3042-3056. https://doi.org/10.1016/j.comnet.2009.07.013nsor. Computer Networks, 53(18), 3042-3056. https://doi.org/10.1016/j.comnet.2009.07.013

Steiner, R. V., \& Lupu, E. (2016). Attestation in Wireless Sensor Networks : A Survey, 49(3), 131.

Umar, I. A., Hanapi, Z. M., Sali, A., \& Zulkarnain, Z. A. (2017). TruFiX: A Configurable TrustBased Cross-Layer Protocol for Wireless Sensor Networks. IEEE Access, 5, 2550-2562. https://doi.org/10.1109/ACCESS.2017.2672827

Usman, A. B., \& Gutierrez, J. (2018). Toward Trust Based Protocols in a Pervasive and Mobile Computing: A Survey. Ad Hoc Networks. https://doi.org/10.1016/j.adhoc.2018.07.009

Vasudeva, A., \& Sood, M. (2018a). Survey on sybil attack defense mechanisms in wireless ad hoc networks. Journal of Network and Computer Applications, 120(June), 78-118. https://doi.org/10.1016/j.jnca.2018.07.006

Vasudeva, A., \& Sood, M. (2018b). Survey on sybil attack defense mechanisms in wireless ad hoc networks. Journal of Network and Computer Applications, 120(June), 78-118. https://doi.org/10.1016/j.jnca.2018.07.006

Wang, C., Zhu, L., Gong, L., Zhao, Z., Yang, L., Liu, Z., \& Cheng, X. (2018). Accurate sybil attack detection based on fine-grained physical channel information. Sensors (Switzerland), 18(3), 1-23. https://doi.org/10.3390/s18030878

Wang, J., Yang, G., Sun, Y., \& Chen, S. (2007). Sybil attack detection based on RSSI for wireless sensor network. 2007 International Conference on Wireless Communications, Networking and Mobile Computing, WiCOM 2007, (06), 2684-2687. https://doi.org/10.1109/WICOM.2007.667

Wen, M., Li, H., Zheng, Y., \& Chen, K. (2008). TDOA-based Sybil attack detection scheme for wireless sensor networks. Journal of Shanghai University (English Edition), 12(1), 66-70. https://doi.org/10.1007/s11741-008-0113-2

Wu, Z., \& Ma, R. (2019). A Novel Sybil Attack Detection Scheme Based on Edge Computing for Mobile IoT Environment. Retrieved from http://arxiv.org/abs/1911.03129

Yuan, Y., Huo, L., Wang, Z., \& Hogrefe, D. (2018). Secure APIT Localization Scheme Against Sybil Attacks in Distributed Wireless Sensor Networks. IEEE Access, 6, 27629-27636. https://doi.org/10.1109/ACCESS.2018.2836898

Zeb, A., Islam, A. K. M. M., Zareei, M., Mamoon, I. Al, \& Mansoor, N. (2016). Clustering Analysis in Wireless Sensor Networks : An ambit of Performance Metrics and Schemes Taxonomy, 1-48.

Zhan, G., Shi, W., \& Deng, J. (2012). Design and implementation of TARF: A trust-aware

Peer] Comput. Sci. reviewing PDF | (CS-2020:10:54560:1:0:NEW 18 May 2021) 
953

954

955

956

957

958

959

960

961

962

963

964

965

966

967

968

969

970

971

972

973

974

975 routing framework for WSNs. IEEE Transactions on Dependable and Secure Computing, 9(2), 184-197. https://doi.org/10.1109/TDSC.2011.58

Zhang, Q., \& Zhou, X. (2010). Efficient distributed location verification in wireless sensor networks. Frontiers of Computer Science in China, 4(1), 123-134. https://doi.org/10.1007/s11704-009-0071-X

Zhong, S., Li, L., Liu, Y. G., \& Yang, Y. R. (2004). Privacy-preserving locationbased services for mobile users in wireless networks. Yale Computer Science, Tech. Rep. YALEU/DCS/TR1297, 1-13. Retrieved from $\mathrm{http} / / /$ scholar.google.com/scholar?hl=en\&btnG=Search\&q=intitle:PrivacyPreserving+Location-based+Services+for+Mobile+Users + in+Wireless + Networks \#0

Zhou, P., Jiang, S., Irissappane, A., Zhang, J., Zhou, J., \& Teo, J. C. M. (2015). Toward energyefficient trust system through watchdog optimization for WSNs. IEEE Transactions on Information Forensics and Security, 10(3), 613-625. https://doi.org/10.1109/TIFS.2015.2389145

Zhu, M., Chang, L., Wang, N., \& You, I. (2020). A smart collaborative routing protocol for delay sensitive applications in industrial IoT. IEEE Access, 8, 20413-20427. https://doi.org/10.1109/ACCESS.2019.2963723

Zhu, S., Setia, S., \& Jajodia, S. (2006). LEAP+: Efficient security mechanisms for large-scale distributed sensor networks. ACM Transactions on Sensor Networks, 2(4), 500-528. https://doi.org/10.1145/1218556.1218559

Zhuang, T., Ren, M., Gao, X., Dong, M., Huang, W., \& Zhang, C. (2019). Insulation Condition Monitoring in Distribution Power Grid via IoT-Based Sensing Network, 34(4), 1706-1714. 


\section{Table $\mathbf{1}$ (on next page)}

Existing literature review on Sybil Attack countermeasures 
1

\begin{tabular}{|c|c|c|c|c|c|c|c|c|c|c|}
\hline Reference & $\begin{array}{c}\text { Key } \\
\text { management }\end{array}$ & $\begin{array}{c}\text { Resource } \\
\text { Testing }\end{array}$ & Encryption & Trust & RSSI & Watchdog & TDOA & Metahueristic & $\begin{array}{c}\text { Incentive } \\
\text { based }\end{array}$ & $\begin{array}{c}\text { Application } \\
\text { Domain }\end{array}$ \\
\hline $\begin{array}{l}\text { This } \\
\text { paper }\end{array}$ & $\checkmark$ & $\checkmark$ & $\checkmark$ & $\checkmark$ & $\checkmark$ & $\checkmark$ & $\checkmark$ & $\checkmark$ & $x$ & WSN \\
\hline $\begin{array}{l}\text { (Vasudeva } \\
\text { \& Sood, } \\
2018 \text { ) }\end{array}$ & $\checkmark$ & $\checkmark$ & $\checkmark$ & $\checkmark$ & $\checkmark$ & $\checkmark$ & $\checkmark$ & $x$ & $x$ & WANET \\
\hline $\begin{array}{l}\text { (Bhise \& } \\
\text { Kamble, } \\
2016 \text { ) }\end{array}$ & $\checkmark$ & $\checkmark$ & $x$ & $x$ & $\checkmark$ & $x$ & $x$ & $X$ & $\checkmark$ & $\begin{array}{c}\text { Social } \\
\text { Network \& } \\
\text { WSN }\end{array}$ \\
\hline $\begin{array}{l}\text { (Gunturu, } \\
\text { 2015) }\end{array}$ & $\checkmark$ & $\checkmark$ & $\mathrm{x}$ & $\checkmark$ & $\mathrm{X}$ & $\mathrm{X}$ & $\mathrm{x}$ & $\mathrm{X}$ & $x$ & $\begin{array}{l}\text { Social } \\
\text { Network }\end{array}$ \\
\hline
\end{tabular}




\section{Table 2 (on next page)}

\section{Research Questions}




\begin{tabular}{lll}
\hline RQ\# & Research questions & Motivations \\
\hline RQ1 & What is Sybil and Blackhole Attack? & $\begin{array}{l}\text { These two questions will prove the aim } \\
\text { of countermeasure for Sybil and } \\
\text { Blackhole attack }\end{array}$ \\
\hline RQ2 & $\begin{array}{l}\text { Why is it to focus on these two attacks in } \\
\text { WSN and IoT environment? }\end{array}$ & $\begin{array}{l}\text { These two questions prove the } \\
\text { purpose of countermeasure for Sybil } \\
\text { and Blackhole attack }\end{array}$ \\
\hline RQ3 & $\begin{array}{l}\text { Where will new researchers concentrate on } \\
\text { creating a new method? }\end{array}$ & $\begin{array}{l}\text { This question is intended to help } \\
\text { researchers look deeper into the } \\
\text { research issue }\end{array}$ \\
& $\begin{array}{l}\text { How can the countermeasures in the Sybil } \\
\text { and Blackhole attain better algorithms to } \\
\text { thwart these attacks? }\end{array}$ & $\begin{array}{l}\text { This question is intended to explain } \\
\text { countermeasure use to thwart Sybil } \\
\text { and Blackhole attack in obtaining } \\
\text { optimal algorithms, identifying } \\
\text { challenges and techniques. }\end{array}$
\end{tabular}




\section{Table $\mathbf{3}$ (on next page)}

Sybil attack countermeasures comparison 


\begin{tabular}{|c|c|c|c|c|c|c|c|c|}
\hline \multirow[t]{2}{*}{ Author, Year } & \multirow[t]{2}{*}{ Advantages } & \multirow[t]{2}{*}{ Disadvantages } & \multirow{2}{*}{$\begin{array}{l}\text { Countermeasure } \\
\text { Method }\end{array}$} & \multicolumn{3}{|c|}{ Type of Countermeasure } & \multirow{2}{*}{$\begin{array}{c}\text { IoT } \\
\text { Ready }\end{array}$} & \multirow[t]{2}{*}{ Simulator } \\
\hline & & & & Prevention & Detection & Mitigation & & \\
\hline $\begin{array}{l}\text { García-Otero } \\
\text { et al. }(2010)\end{array}$ & Energy efficient & $\begin{array}{c}\text { Communication } \\
\text { overhead }\end{array}$ & Trust & & $\checkmark$ & & & $\begin{array}{l}\text { AWISSENET } \\
\text { test-bed. }\end{array}$ \\
\hline $\begin{array}{l}\text { Zhang et al. } \\
\text { (2010) }\end{array}$ & $\begin{array}{l}\text { low false positive rates } \\
\text { at the same time even } \\
\text { with high location } \\
\text { inaccuracy }\end{array}$ & $\begin{array}{l}\text { High } \\
\text { communication } \\
\text { overhead due to } \\
\text { exchange of trust } \\
\text { information }\end{array}$ & $\begin{array}{l}\text { Encryption and } \\
\text { Trust }\end{array}$ & $\checkmark$ & $\checkmark$ & & $\checkmark$ & $\begin{array}{l}\text { Mathematical } \\
\text { proof }\end{array}$ \\
\hline $\begin{array}{l}\text { Claycomb, et } \\
\text { al. (2011) }\end{array}$ & $\begin{array}{l}\text { A new approach to key } \\
\text { establishment, which } \\
\text { combines a group- } \\
\text { based distribution } \\
\text { model and identity- } \\
\text { based cryptography }\end{array}$ & High complexity & Encryption & $\checkmark$ & & & & NA \\
\hline He et al.(2011) & $\begin{array}{l}\text { EDDK utilizes } \\
\text { encryption method is } \\
\text { more advantageous in } \\
\text { computation, } \\
\text { communication, and } \\
\text { storage }\end{array}$ & High computation & Encryption & $\checkmark$ & & & & MATLAB \\
\hline $\begin{array}{l}\text { Dong et al. } \\
\text { (2012) }\end{array}$ & $\begin{array}{l}\text { Extend the lifetime of } \\
\text { the whole network. }\end{array}$ & $\begin{array}{l}\text { Does not support } \\
\text { dynamic regular } \\
\text { sensor node } \\
\text { addition after initial } \\
\text { deployment }\end{array}$ & Encryption & $\checkmark$ & & & & TelosB motes \\
\hline $\begin{array}{l}\text { Zhan et al. } \\
\text { (2012) }\end{array}$ & $\begin{array}{l}\text { TARF focuses on } \\
\text { trustworthiness and } \\
\text { energy efficiency, } \\
\text { which are vital to the } \\
\text { survival of a WSN in a } \\
\text { hostile environment }\end{array}$ & $\begin{array}{c}\text { TARF is suitable } \\
\text { only for static } \\
\text { environment }\end{array}$ & Trust & & $\checkmark$ & & $\checkmark$ & MATLAB \\
\hline $\begin{array}{l}\text { Kim et al. } \\
(2013)\end{array}$ & $\begin{array}{l}\text { Less storage, } \\
\text { computation and } \\
\text { communication cost }\end{array}$ & High processing & Encryption & $\checkmark$ & & & & $\begin{array}{l}\text { Mathematical } \\
\text { proof }\end{array}$ \\
\hline $\begin{array}{l}\text { Lyu et al. } \\
(2015)\end{array}$ & $\begin{array}{l}\text { Lightweight and } \\
\text { distributed }\end{array}$ & $\begin{array}{c}\text { High } \\
\text { communication }\end{array}$ & Trust \& RSSI & $\checkmark$ & $\checkmark$ & & $\checkmark$ & OPNET \\
\hline
\end{tabular}




\begin{tabular}{|c|c|c|c|c|c|c|c|c|}
\hline & & load & & & & & & \\
\hline $\begin{array}{l}\text { Sarigiannidis } \\
\text { et al. (2015) }\end{array}$ & $\begin{array}{l}\text { Lightweight and } \\
\text { distributed }\end{array}$ & $\begin{array}{l}\text { Mobility is not } \\
\text { considered and not } \\
\text { energy efficient }\end{array}$ & $\begin{array}{l}\text { Using UWB } \\
\text { antenna dan } \\
\text { revoke malicious } \\
\text { when detected } \\
\end{array}$ & & $\checkmark$ & $\checkmark$ & & MATLAB \\
\hline $\begin{array}{l}\text { Zhou et al. } \\
\text { (2015) }\end{array}$ & $\begin{array}{l}\text { Lightweight and } \\
\text { energy efficient }\end{array}$ & $\begin{array}{c}\text { High } \\
\text { communication } \\
\text { overhead }\end{array}$ & $\begin{array}{l}\text { Watchdog using } \\
\text { Trust }\end{array}$ & & $\checkmark$ & & & WSNET \\
\hline $\begin{array}{l}\text { Jan et al. } \\
\text { (2015) }\end{array}$ & $\begin{array}{l}\text { Improve network } \\
\text { lifetime }\end{array}$ & $\begin{array}{l}\text { The system fails if a } \\
\text { malicious node } \\
\text { able to imitate high } \\
\text { energy node }\end{array}$ & RSSI & & $\checkmark$ & & $\checkmark$ & NA \\
\hline $\begin{array}{l}\text { Saleem et al. } \\
\text { (2016) }\end{array}$ & $\begin{array}{l}\text { Provide security } \\
\text { through encryption } \\
\text { with minimum } \\
\text { processing time }\end{array}$ & $\begin{array}{l}\text { Artificial immune } \\
\text { system (AIS) - he } \\
\text { major limitation of } \\
\text { BIOSARP is that it } \\
\text { requires time to } \\
\text { develop the } \\
\text { knowledge of the } \\
\text { overall network } \\
\text { during the } \\
\text { initialization } \\
\text { decryption - } \\
\text { computational } \\
\text { overhead }\end{array}$ & Encryption & $\checkmark$ & & & $\checkmark$ & NS2 \\
\hline $\begin{array}{l}\text { Alsaedi et al. } \\
\text { (2017) }\end{array}$ & $\begin{array}{c}\text { Lightweight and able } \\
\text { to detect Sybil nodes } \\
\text { accurately }\end{array}$ & $\begin{array}{l}\text { Higher memory } \\
\text { requirement }\end{array}$ & $\begin{array}{r}\text { Energy } \\
\text { trust calculation }\end{array}$ & & $\checkmark$ & & $\checkmark$ & $\mathrm{OMNeT}++$ \\
\hline $\begin{array}{l}\text { Prathusha et al. } \\
\text { (2017) }\end{array}$ & $\begin{array}{l}\text { Higher security due to } \\
\text { the encryption method }\end{array}$ & $\begin{array}{c}\text { Higher processing } \\
\text { resources }\end{array}$ & Encryption & $\checkmark$ & & & $\checkmark$ & NetTopo \\
\hline $\begin{array}{l}\text { Raja et al. } \\
(2017)\end{array}$ & $\begin{array}{l}\text { Higher security due to } \\
\text { the encryption method }\end{array}$ & $\begin{array}{l}\text { Higher processing } \\
\text { resources }\end{array}$ & $\begin{array}{c}\text { Encryption using } \\
\text { Fujisaki Okamoto } \\
\text { Algorithm } \\
\end{array}$ & $\checkmark$ & & & & NS2 \\
\hline $\begin{array}{l}\text { Maddar et al. } \\
\text { (2017) }\end{array}$ & Lightweight & $\begin{array}{c}\text { High } \\
\text { communication } \\
\text { overload }\end{array}$ & Trust calculation & & $\checkmark$ & & & MATLAB \\
\hline $\begin{array}{l}\text { Razaque et al. } \\
\text { (2017) }\end{array}$ & $\begin{array}{l}\text { Higher security due to } \\
\text { the encryption method }\end{array}$ & $\begin{array}{l}\text { Higher processing } \\
\text { resources }\end{array}$ & Encryption & $\checkmark$ & & & & NS3 \\
\hline $\begin{array}{l}\text { Yuan et al. } \\
(2018)\end{array}$ & Lightweight & $\begin{array}{l}\text { Not reliable due to } \\
\text { radio interference }\end{array}$ & $\begin{array}{c}\text { Localization with } \\
\text { RSS signal } \\
\end{array}$ & & $\checkmark$ & & & MATLAB \\
\hline
\end{tabular}




\begin{tabular}{|c|c|c|c|c|c|c|c|}
\hline & & $\begin{array}{c}\text { and signal } \\
\text { propagation }\end{array}$ & & & & & \\
\hline $\begin{array}{l}\text { Wang et al. } \\
\text { (2018) }\end{array}$ & $\begin{array}{l}\text { Sybil attack detection } \\
\text { system achieves high } \\
\text { accuracy for both static } \\
\text { and dynamic scenarios } \\
\text { using CSI }\end{array}$ & $\begin{array}{l}\text { It is not easily } \\
\text { obtainable on the } \\
\text { shelf NICs }\end{array}$ & CSI \& RSSI & & $\checkmark$ & & MATLAB \\
\hline $\begin{array}{l}\text { Jamshidi et al. } \\
\text { (2019) }\end{array}$ & Lightweight & $\begin{array}{l}\text { Not reliable due to } \\
\text { radio interference } \\
\text { and signal } \\
\text { propagation }\end{array}$ & RSSI & $\checkmark$ & $\checkmark$ & $\checkmark$ & J-SIM \\
\hline Li et al. (2019) & $\begin{array}{c}\text { Higher accuracy and } \\
\text { able to detect Sybil } \\
\text { node }\end{array}$ & $\begin{array}{l}\text { High processing } \\
\text { load and } \\
\text { communication } \\
\text { overhead }\end{array}$ & $\begin{array}{c}\text { Channel feature in } \\
\text { terms of power } \\
\text { gain and delay } \\
\text { spread. } \\
\end{array}$ & & $\checkmark$ & & MATLAB \\
\hline $\begin{array}{l}\text { Liu et al. } \\
\text { (2020) }\end{array}$ & $\begin{array}{l}\text { Rapid localization } \\
\text { capability and high } \\
\text { precision detection } \\
\text { with low energy } \\
\text { consumption. }\end{array}$ & $\begin{array}{l}\text { Not reliable due to } \\
\text { radio interference } \\
\text { and signal } \\
\text { propagation }\end{array}$ & RSSI & & $\checkmark$ & $\checkmark$ & - \\
\hline $\begin{array}{l}\text { Angappan et } \\
\text { al. (2020) }\end{array}$ & $\begin{array}{l}\text { Can apply to any } \\
\text { resource-constrained } \\
\text { WSN }\end{array}$ & $\begin{array}{c}\text { It takes up memory } \\
\text { if the device has } \\
\text { limited }\end{array}$ & RSSI & & $\checkmark$ & $\checkmark$ & NS2 \\
\hline $\begin{array}{l}\text { Prithi et al. } \\
(2020)\end{array}$ & $\begin{array}{l}\text { Better throughput and } \\
\text { network lifetime }\end{array}$ & $\begin{array}{l}\text { PSO easily to fall } \\
\text { into local optimum } \\
\text { and low } \\
\text { convergence rate in } \\
\text { the iterative } \\
\text { process. .. }\end{array}$ & $\begin{array}{c}\text { Learning } \\
\text { Dynamic } \\
\text { Deterministic } \\
\text { Finite Automata }\end{array}$ & & $\checkmark$ & & MATLAB \\
\hline $\begin{array}{l}\text { Jain, Hussain } \\
\text { et al. }(2020)\end{array}$ & $\begin{array}{l}\text { Hence, the proposed } \\
\text { mechanism is best } \\
\text { suitable for resource } \\
\text { constraint nodes }\end{array}$ & $\begin{array}{c}\text { Hierarchical } \\
\text { network topology } \\
\text { suffers from non- } \\
\text { uniform clustering, } \\
\text { high energy } \\
\text { dissipation, and less } \\
\text { lifespan of the } \\
\text { sensor nod }\end{array}$ & Authentication & $\checkmark$ & & & $\begin{array}{l}\text { AVISPA and } \\
\text { Scyther tools }\end{array}$ \\
\hline
\end{tabular}




\begin{tabular}{|c|c|c|c|c|c|c|c|}
\hline $\begin{array}{l}\text { Giri et al } \\
(2020)\end{array}$ & $\begin{array}{l}\text { Successfully detect } \\
\text { sybil attack and } \\
\text { increase localization } \\
\text { accuracy despite sybil } \\
\text { attack. }\end{array}$ & $\begin{array}{l}\text { High processing } \\
\text { requirement }\end{array}$ & $\begin{array}{l}\text { Localization with } \\
\text { RSS signal }\end{array}$ & & $\checkmark$ & & na \\
\hline $\begin{array}{l}\text { Raghav et al. } \\
(2020)\end{array}$ & $\begin{array}{l}\text { Beeware based secure } \\
\text { and optimized routing } \\
\text { scheme with the help } \\
\text { of bee algorithms. }\end{array}$ & $\begin{array}{l}\text { High processing } \\
\text { requirement \& } \\
\text { moderate scalability } \\
\text { Its disadvantages } \\
\text { include the } \\
\text { diversity of race is } \\
\text { poor. When the } \\
\text { solution is close to } \\
\text { the global } \\
\text { optimum, it is easy } \\
\text { to fall into the local } \\
\text { optimum, resulting } \\
\text { in a stagnation } \\
\text { phenomenon }\end{array}$ & $\begin{array}{l}\text { Encryption \& Bee } \\
\text { Algorithm }\end{array}$ & $\checkmark$ & & $\checkmark$ & MATLAB \\
\hline $\begin{array}{l}\text { Bhushan et al. } \\
(2020)\end{array}$ & $\begin{array}{l}\text { Enhances energy } \\
\text { efficiency and makes } \\
\text { the network secure }\end{array}$ & $\begin{array}{c}\text { Does not cover for } \\
\text { availability }\end{array}$ & Trust and ACO & & $\checkmark$ & & NA \\
\hline
\end{tabular}


Figure 1

The selection of process of articles in the form of diagram 


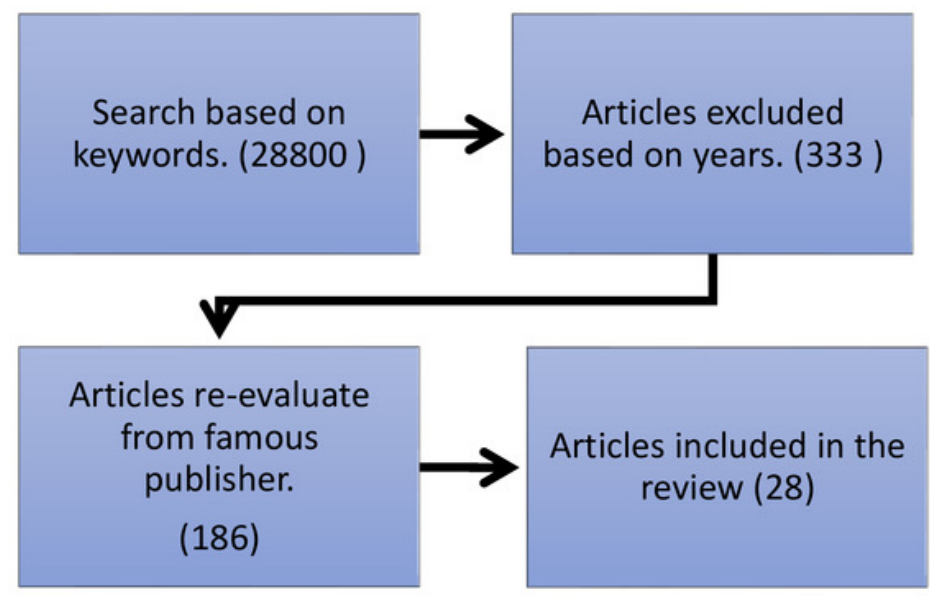


Figure 2

The classification of Attack in WSN and IoT 


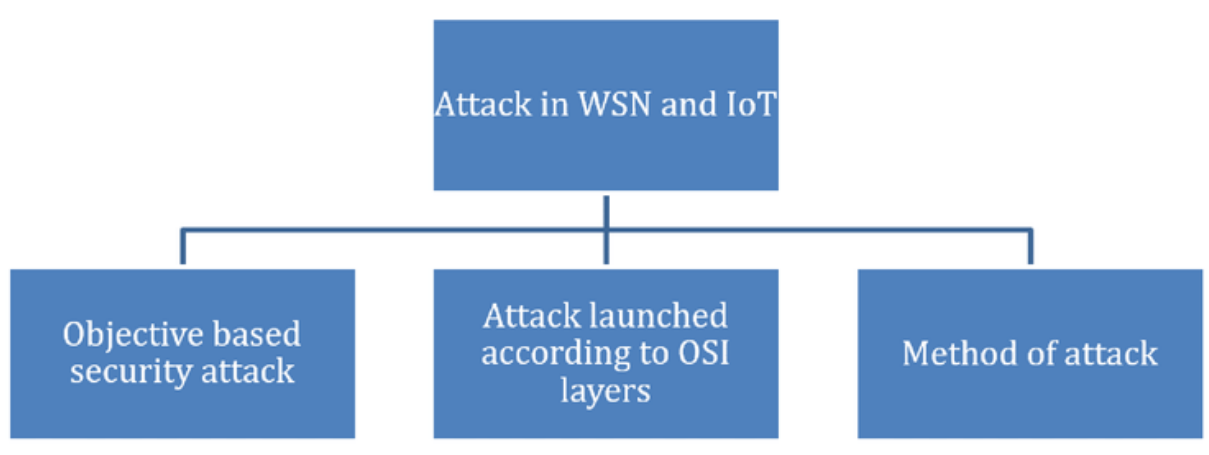


Figure 3

Sybil attack countermeasures framework 


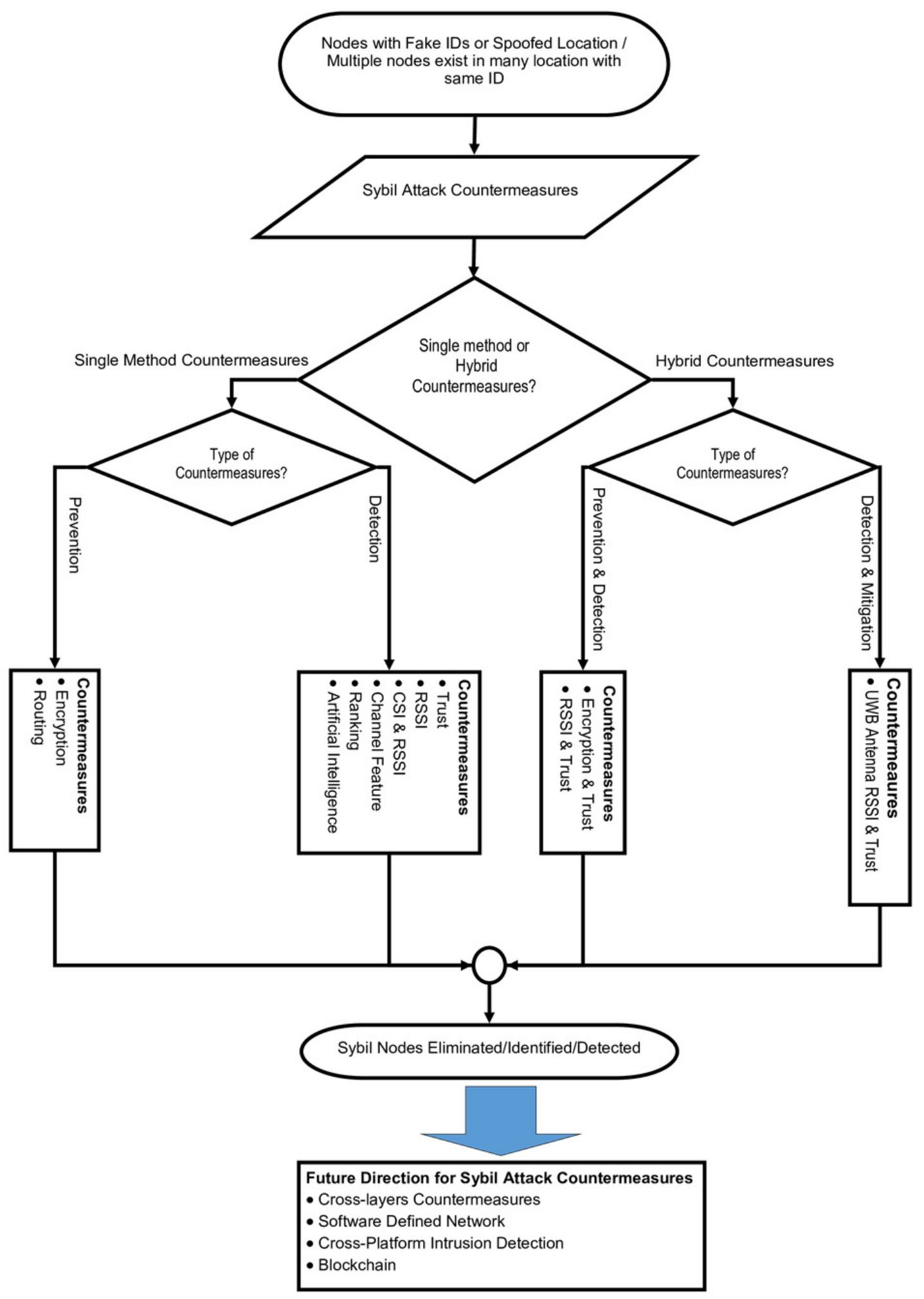


Figure 4

The Sybil attack in geographic routing 


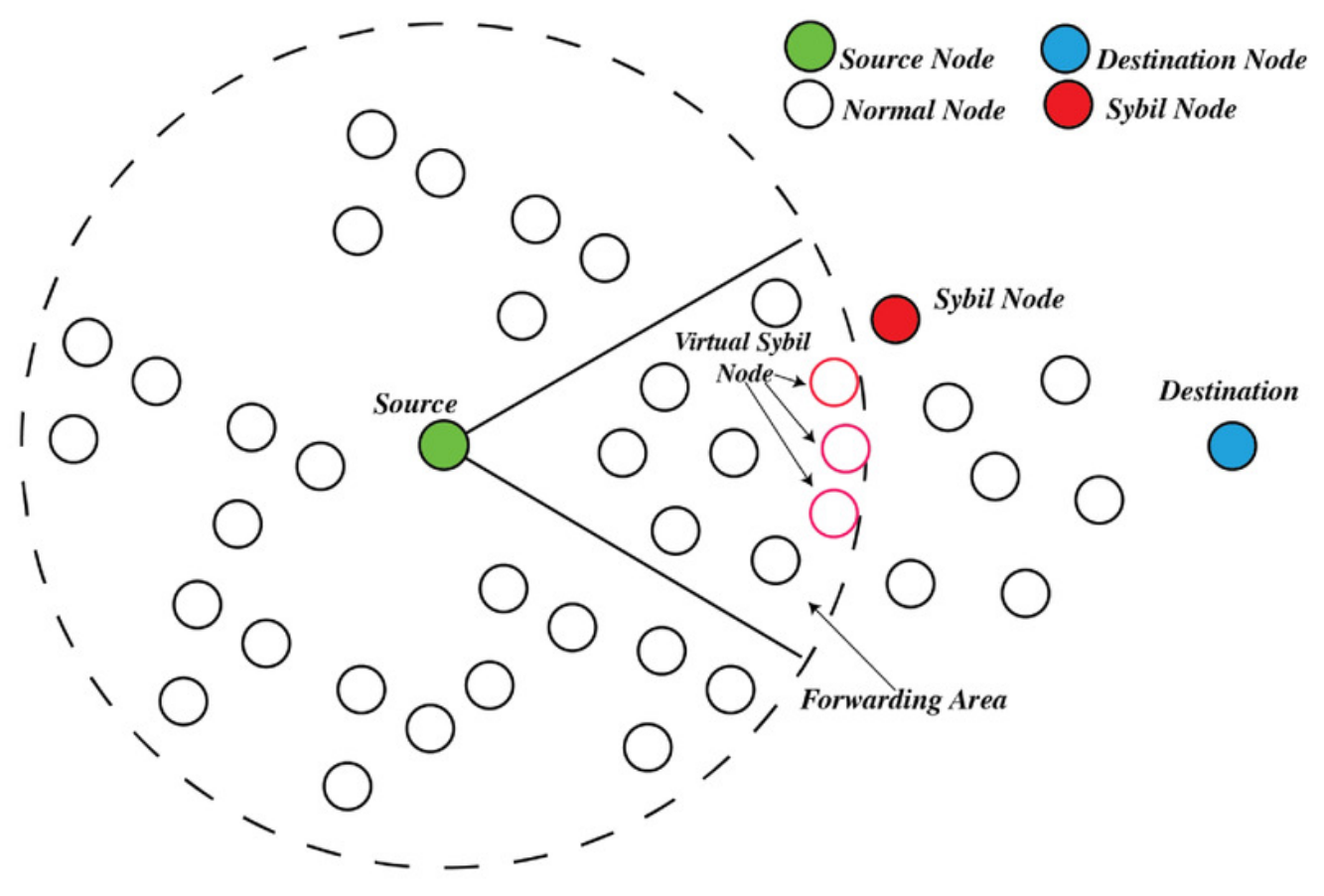


Figure 5

Distribution of the Sybil attack countermeasures between 2010 and 2010 


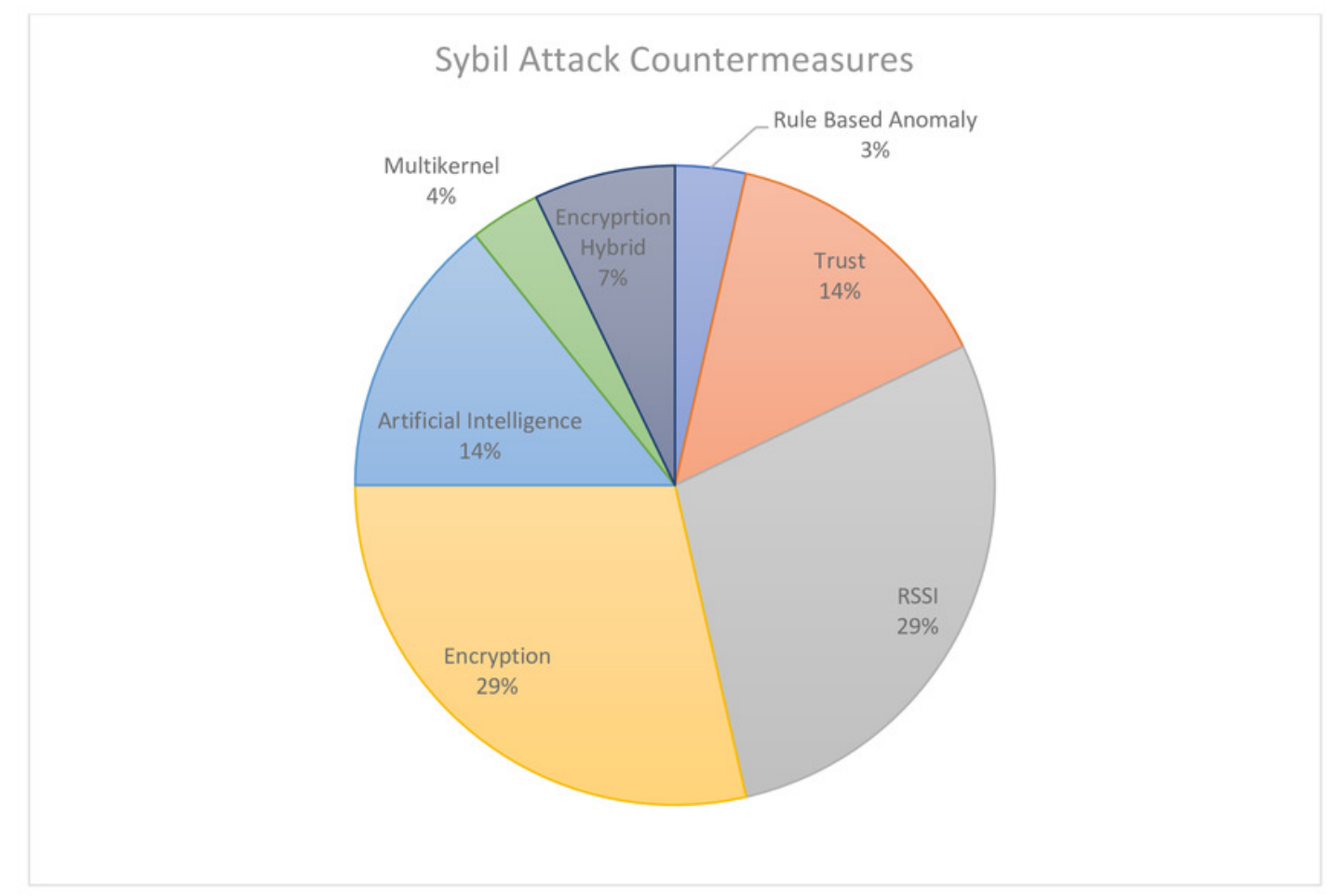


Figure 6

Number of papers on Sybil Countermeasures between 2010 and 2020 


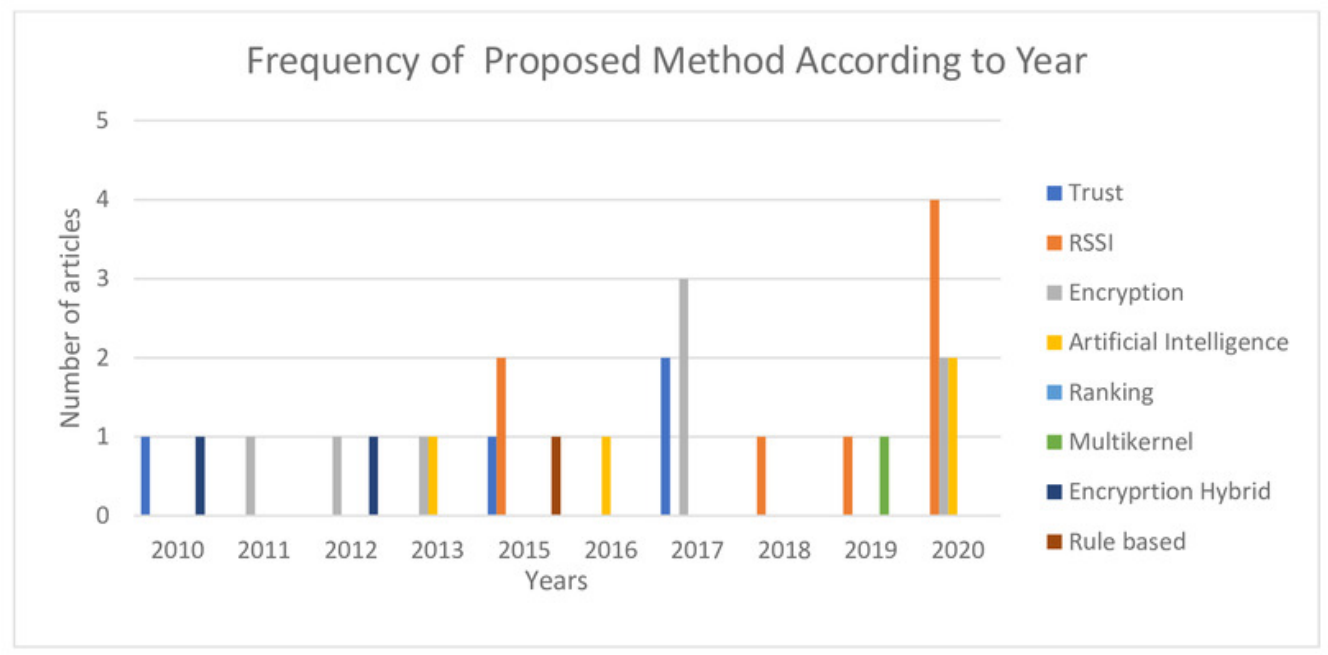

